\title{
Constraining the Circumbinary Disk Tilt in the KH 15D system
}

\author{
Michael Poon, ${ }^{1,2 \star}$ J. J. Zanazzi, ${ }^{1} \dagger$ and Wei Zhu, ${ }^{1,3}{ }^{\dagger}$ \\ ${ }^{1}$ Canadian Institute for Theoretical Astrophysics, University of Toronto, 60 St. George Street, Toronto, Ontario, M5S 1A7, Canada \\ ${ }^{2}$ Department of Astronomy and Astrophysics, University of Toronto, Toronto, ON M5S 3H4, Canada \\ ${ }^{3}$ Department of Astronomy, Tsinghua University, Beijing, 100084, China
}

Accepted XXX. Received YYY; in original form ZZZ

\begin{abstract}
$\mathrm{KH} 15 \mathrm{D}$ is a system which consists of a young, eccentric binary, and a circumbinary disk which obscures the binary as the disk precesses. We develop a self-consistent model that provides a reasonable fit to the photometric variability that was observed in the KH 15D system over the past 60 years. Our model suggests that the circumbinary disk has an inner edge $r_{\text {in }} \lesssim 1$ au, an outer edge $r_{\text {out }} \sim$ a few au, and that the disk is misaligned relative to the stellar binary by $\sim 5-16$ degrees, with the inner edge more inclined than the outer edge. The difference between the inclinations (warp) and longitude of ascending nodes (twist) at the inner and outer edges of the disk are of order $\sim 10$ degrees and $\sim 15$ degrees, respectively. We also provide constraints on other properties of the disk, such as the precession period and surface density profile. Our work demonstrates the power of photometric data in constraining the physical properties of planet-forming circumbinary disks.
\end{abstract}

Key words: protoplanetary discs - planets and satellites: formation - stars: individual (KH 15D) - binaries: spectroscopic techniques: photometric

\section{INTRODUCTION}

Our understanding of tilts within planet-forming circumbinary systems has undergone drastic changes within the past decade. Originally, the basic picture was quite simple: a circumbinary disk should always be observed to be aligned with the orbital plane of the binary. Even though simulations of turbulent molecular clouds found circumbinary disks frequently formed misaligned with the orbital plane of the binary (e.g. Bate et al. 2002; Bate 2012, 2018), viscous disk-warping torques were showed to damp the disk-binary inclination over timescales much shorter than typical protoplanetary disk lifetimes (Foucart \& Lai 2013, 2014). Most inclination constraints on protoplanetary (e.g. Andrews et al. 2010; Rosenfeld et al. 2012; Czekala et al. 2015, 2016; Ruíz-Rodríguez et al. 2019) and debris (e.g. Kennedy et al. 2012b) disks confirm this basic picture, finding alignment of the disk with the orbital plane of the binary to within a few degrees.

However, after the detection of a few highly inclined circumbinary disks (e.g. Kennedy et al. 2012a; Marino et al. 2015; Brinch et al. 2016; Czekala et al. 2017), it became clear not all circumbinary disks align rapidly. Motivated by these detections of highly-inclined disks, the theoretical community found that when a circumbinary disk orbits an eccentric binary, the disk-binary inclination can grow under certain circumstances, evolving eventually to $90^{\circ}$ (polar alignment; Aly et al. 2015; Martin \& Lubow 2017; Zanazzi \& Lai 2018). Additional inclined circumbinary disks orbiting eccentric binaries were discovered soon thereafter, such as HD 98800 (Kennedy et al. 2019),

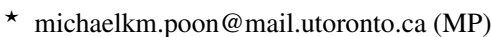

† jzanazzi@cita.utoronto.ca (JJZ)

†eizhu@cita.utoronto.ca (WZ)
}

and AB Aurigae (Poblete et al. 2020). Recently, Czekala et al. (2019) showed circumbinary disks had larger inclinations when orbiting binaries with higher eccentricities, further supporting the operation of this mechanism in circumbinary disk systems.

The alignment process itself was also shown to be non-trivial, with the disk itself occasionally breaking in the process. Early on, the disk was expected to remain nearly flat, due to the resonant propagation of bending waves across the disk (Papaloizou \& Lin 1995; Lubow \& Ogilvie 2000). Later hydrodynamical simulations found that the disk under some circumstances may break, with different disk annuli becoming highly misaligned with one another, due to strong differential nodal precession induced by the torque from the binary (e.g. Nixon et al. 2013; Facchini et al. 2013). Numerous broken protoplanetary disks orbiting two binary stars have subsequently been found, including HD 142527 (Marino et al. 2015; Price et al. 2018) and GW Ori (Bi et al. 2020; Kraus et al. 2020).

The inclinations of detected circumbinary planets, in contrast, remain broadly consistent with formation in nearly-aligned circumbinary disks. After the detection of a few dozen circumbinary planets (see Welsh \& Orosz 2018; Doyle \& Deeg 2018 for recent reviews), the inclinations within the circumbinary planet population are consistent with alignment to within $\sim 4^{\circ}$ (Martin \& Triaud 2014; Armstrong et al. 2014; Li et al. 2016). However, highly-misaligned circumbinary planets are physically allowed, because the inclined orbit has been shown to be long-term stable once a planet forms in the polaraligned circumbinary disk. (Doolin \& Blundell 2011; Giuppone \& Cuello 2019; Chen et al. 2019, 2020). New detection methods may detect polar-aligned circumbinary planets in the future (Zhang \& Fabrycky 2019).

While a large number of systems now have constraints on mutual 
inclinations between the disk and the binary orbital planes, there remain few constraints on twists and warps within the circumbinary disk itself. This is because the methods used to constrain disk inclinations are not sensitive to the small misalignments within the disk. Gaseous protoplanetary disk inclinations are constrained via the orbital motion of the disk gas through the Doppler shift of emission lines (e.g. Facchini et al. 2018; Price et al. 2018). Debris disk inclinations are constrained by the orientation of the disk implied by its continuum emission (e.g. Kennedy et al. 2012a,b).

A rare example of a circumbinary disk $^{1}$ system where photometric constraints exist is Kearns-Herbst 15D (KH 15D) (Kearns \& Herbst 1998). KH $15 \mathrm{D}$ is a system with a highly-unusual light curve, which exhibited dips by up to 5 magnitudes. The morphology of the dipping behavior changed over decade-long timescales, but displayed periodicity over short 48 day timescales. The complex light curve of this system is generally believed to be due to a circumbinary disk and a binary star, with some of the dips caused by the optically thick, precessing disk slowly and obscuring the orbital plane of the stellar binary (Winn et al. 2004, 2006; Chiang \& Murray-Clay 2004; Capelo et al. 2012). Although much work has gone into understanding $\mathrm{KH}$ $15 \mathrm{D}$, no work has attempted to provide quantitative constraints on the properties of the warped disk based on the photometric data.

In this work, we combine the spectroscopic and photometric data to constrain the properties of the circumbinary disk KH 15D. With recent data up to 2018 from Aronow et al. (2018) and García Soto et al. (2020), we improve the Winn et al. (2006) model to fit all photometric data since 1955 . Our results are particularly exciting, as our fit nearly encompasses the full transit of the circumbinary disk of KH 15D. Section 2 extends the Winn et al. (2006) model to fit the light curve of the system, over the more than 60 year duration the system was observed. Section 3 develops a dynamical model to constrain the circumbinary disk properties implied by the photometric constraints. Section 4 discusses the theoretical implications of our work, improvements which can be made to our model, and our model predictions which can be tested with future observations. Section 5 summarizes the conclusions of our work.

\section{MODELLING THE LIGHT CURVE OF KH 15D}

\subsection{Photometric \& Radial Velocity Observations}

We use radial velocity observations to constrain the orbit of the stellar binary and photometric data to constrain the geometry of the optically thick, precessing disk. We begin with a brief description of the radial velocity and photometric data used in this work.

We use the Radial Velocity (RV) measurements gathered by Hamilton et al. (2003) and Johnson et al. (2004), and because one of the stars is occulted by the disk when the RV measurements are taken, this is effectively a single-lined spectroscopic binary. As in Winn et al. (2006), we only use RV measurements gathered when the system flux is $90 \%$ or greater than its mean out-of-occultation flux,

\footnotetext{
${ }^{1}$ In this work, we use the terminology disk, rather than ring, to describe the object (likely) extending only to a few au around the KH 15D stellar binary. Although this runs counter to more traditional ideas of what a protoplanetary disk is within the planet-formation community, which has envisioned a disk as a gaseous object orbiting a young stellar object out to tens or hundreds of au, recent observations have detected more compact protoplanetary disks. Pegues et al. (2021) found the CO emission around the young M-dwarf FP Tau extended to $\sim 4-8$ au, while Francis \& van der Marel (2020) resolved the size of the inner disks in a number of transition disk systems to lie near or within $\sim 1-10$ au.
}

because the Rossiter-McLaughlin effect (Rossiter 1924; McLaughlin 1924) leads to systematic errors as the stellar companion is occulted by the disk ${ }^{2}$. This gives $12 \mathrm{RV}$ measurements to aid in constraining the orbit of the binary.

For the photometry, we use the tabulated data from Winn et al. (2006), Aronow et al. (2018), and García Soto et al. (2020). Details about the photometric observations can be found in these references, and we only provide a brief summary here. These catalogues include data from photographic plates from the 1950s to 1985 (Johnson \& Winn 2004; Maffei et al. 2005), as well as observations using ChargeCoupled Devices (CCDs) since 1995 (e.g. Hamilton et al. 2005; Capelo et al. 2012; Aronow et al. 2018; García Soto et al. 2020). No observations were known to be taken between 1985-1994. All photometric observations have been transformed into the standard Cousin I-band measurements. We bin the original data-set (6241 points) into 2813 data points in order to reduce the amount of time needed for the photometric model computation. The uncertainties of all photometric measurements are re-scaled up by a factor of two, to allow for a model fit which gives a reduced $\chi^{2}$ close to unity.

\subsection{Previous models for KH 15D}

So far four models have been proposed to explain the photometric variation in the $\mathrm{KH} 15 \mathrm{D}$ system. The phenomenological model by Winn et al. (2004, 2006) approximates the leading edge of the disk as an infinitely long and optically thick screen, which occults the two stars as the screen moves across the orbit of the binary. Motivated by dynamics, Chiang \& Murray-Clay (2004) treat the KH 15D disk as a warped disk with finite optical depth, and model the photometric variations by a disk precessing into and out of the line-of-sight of the observer. Silvia \& Agol (2008) developed their model based on the model of Winn et al. (2006), but introduced more disk-related physics, such as the finite optical depth, curvature near the edge, and forward-scattering of starlight from the dust in the disk (which was parameterized as "halos" in the Winn et al. 2006 model). The fourth model is that of García Soto et al. (2020), who extended the Winn et al. (2006) model to include a trailing, as well as leading, edge. We choose to build our model based on Winn et al. (2006), because it allows us to remain agnostic about the detailed physics of the disk itself, while still accurately fitting the light curve of KH 15D. We review the Winn et al. (2006) model within this subsection.

Figure 1 illustrates the physical motivation behind the Winn et al. (2006) model. A single "leading" edge (red dashed line) slowly advances over the orbital plane of the binary, which approximates the inner or outer truncation radius of the disk slowly covering both stars as the disk is precessing around the binary. For data taken before 2005 , it is reasonable to neglect the outer disk truncation radius (the "trailing" edge, as marked by the yellow dashed curve). To model how quickly the leading edge advanced across the orbit of the binary, Winn et al. (2006) used the latest date when star B was still visible $\left(t_{4}\right)$, and the latest date when the orbit of star A was visible $\left(t_{5}\right.$, see Fig. 2 left panel), as free parameters in their model. In addition, not only was the angle the leading edge made with the $X$-axis of the observer allowed to vary, but it was also allowed to change at a constant rate, controlled by two free parameters $\theta_{L}\left(t_{4}\right)$ and $\dot{\theta}_{L}$. The light from the binary was modelled with 7 parameters, with the luminosity from star A (B) denoted by $L_{\mathrm{A}}\left(L_{\mathrm{B}}\right)$, the background light when the disk fully occults the binary by $L_{0}$, with the parameters $\left\{\epsilon_{1}, \epsilon_{2}, \xi_{1}, \xi_{2}\right\}$

\footnotetext{
2 We note that the Rossiter-McLaughlin effect is only important in this sys-
} tem, if the KH 15D disk edges are sharp, not diffuse. 


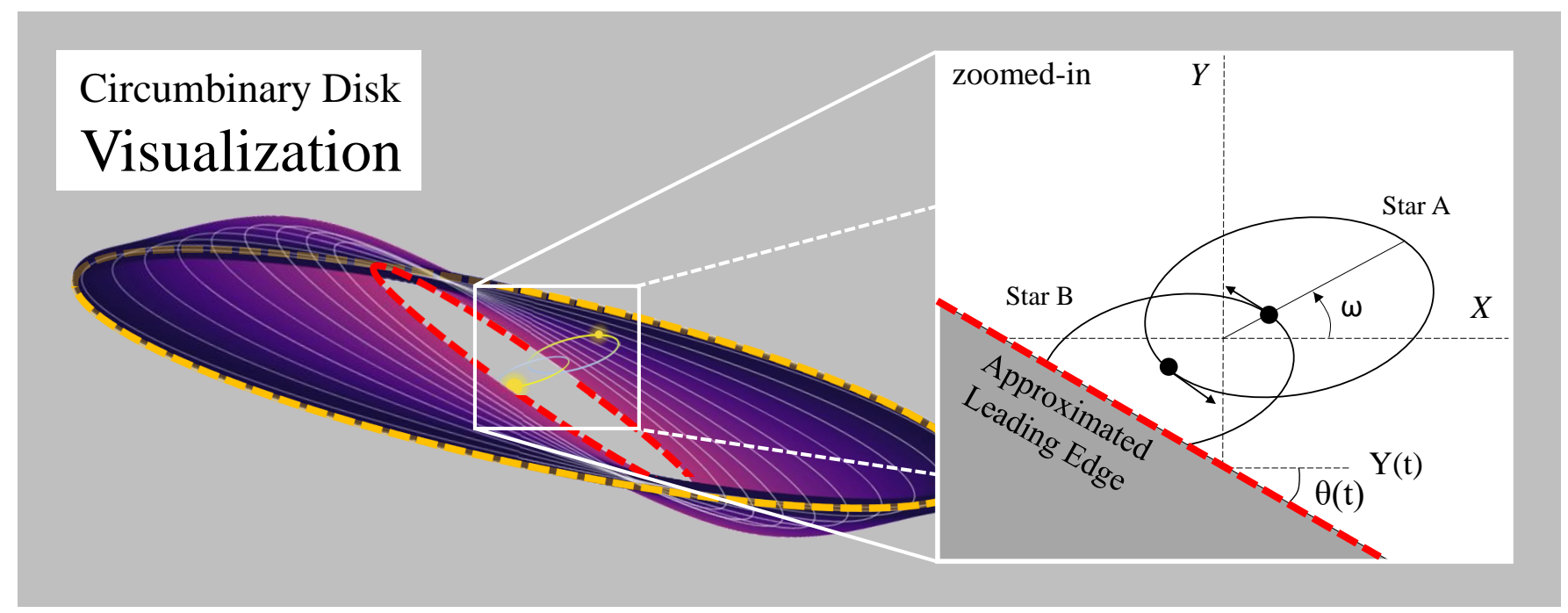

Figure 1. The purple ellipses display a potential shape for the circumbinary disk of KH 15D. No assumption about the inclination between the innermost edge (red dashed curve), outermost edge (yellow dashed curve) or binary plane (centre yellow ellipses) are made while modelling the occultations of KH 15D. We note that our model also allows for the leading edge to be the outermost truncation radius of the disk, with the trailing edge as the innermost truncation radius of the disk. The zoomed-in inset diagram displays how the circumbinary disk geometry occults the binary of KH 15D. The inner or outer truncation radius of the disk slowly covers the orbital plane of the binary, as the disk precesses around the orbital angular momentum axis of the binary. We approximate the inner and outer disk edges as straight edges as the binary is occulted.

parameterizing the light emitted by halos surrounding stars A and B. Specifically, the 1D brightness distribution from star $i=\mathrm{A}, \mathrm{B}$ was taken to be

$B_{i}(v)=\left\{\begin{array}{ll}\left(\epsilon_{1} / \xi_{1}\right) \exp \left[(v+1) / \xi_{1}\right] & v \leq-1 \\ \left(\epsilon_{1} / \xi_{1}\right)+B_{\star i}(v) & -1<v<1 \\ \left(\epsilon_{2} / \xi_{2}\right) \exp \left[-(v-1) / \xi_{2}\right] & v \geq 1\end{array}\right.$,

where $B_{\star i}$ is the $1 \mathrm{D}$ brightness distribution of star $i$, assuming a linear limb-darkening model:

$B_{\star i}(v)=2 I_{i} \sqrt{1-v^{2}}\left[1-u\left(1-\frac{\pi}{4} \sqrt{1-v^{2}}\right)\right]$.

Here, $u=0.65$ is the limb-darkening coefficient for both stars, and $I_{i}$ is the reference intensity of star $i$. Letting $y_{L, i}$ be the distance of the lead edge from star $i$, and $v_{L, i}=y_{L, i} / R_{i}$, then the flux from star $i$ is

$F_{L, i}=\int_{v_{L, i}}^{\infty} B_{i}(v) \mathrm{d} v$

with the total flux $F=F_{L, \mathrm{~A}}+F_{L, \mathrm{~B}}$. Physically, each "halo" parameterizes forward-scattering of starlight by dust in the disk (Winn et al. 2006; Silvia \& Agol 2008). The mass and radius for star A were taken to be $M_{\mathrm{A}}=0.6 \mathrm{M}_{\odot}$ and $R_{\mathrm{A}}=1.3 \mathrm{R}_{\odot}$, while the ratios between the masses and radii of the two stars are $M_{\mathrm{B}} / M_{\mathrm{A}}=1.2$ and $R_{\mathrm{B}} / R_{\mathrm{A}}=1.05$, respectively. The orbit of the binary is described by standard orbital parameters used to model RV data, with an orbital period $P$, eccentricity $e$, inclination $I$, longitude of pericenter $\omega$, time of pericenter passage $T_{p}$, and line-of-sight velocity $\gamma$ (see e.g. Fulton et al. 2018 for details). The Cartesian coordinate system in the sky-projected reference plane of the observer $(X, Y)$ is chosen so the $X$-axis lies along the line of nodes (so $\Omega=0$ ).

The best fit model parameters for the KH $15 \mathrm{D}$ system was then calculated by minimizing (Winn et al. 2004, 2006)

$$
\begin{aligned}
\chi^{2} & =\sum_{j=1}^{N_{F}}\left(\frac{F_{j}-F_{O, j}}{\sigma_{F, j}}\right)^{2}+\lambda \sum_{j=1}^{N_{V}}\left(\frac{V_{j}-V_{O, j}}{\sigma_{V, j}}\right)^{2} \\
& \equiv \chi_{\text {phot }}^{2}+\lambda \chi_{\mathrm{RV}}^{2},
\end{aligned}
$$

where $\chi_{\mathrm{phot}}^{2}$ is the $\chi^{2}$ of the photometry model alone, $\chi_{\mathrm{RV}}^{2}$ is the $\chi^{2}$ metric of the modelled orbit of the binary in relation to the RV data (see Fulton et al. 2018 for details), and for a quantity $X, X_{j}$ denotes the model prediction at point $j, X_{O, j}$ denotes the observed value of $X$ at $j$, while $\sigma_{X, j}$ denotes the uncertainty of $X_{O, j}$ at $j$. The parameter $\lambda=50$ was chosen to increase the importance of the RV model relative to that for the photometry, because the model constraining the orbit of the binary (a Keplerian orbit) is much more certain than the model describing the light curve of the binary (occulted by a precessing disk).

Because the screen advances in the positive vertical direction at a constant rate, an equivalent way of parameterizing the ascent of the screen are through where the screen intersects the $Y$-axis at the orbital contact time $t_{4}$, which we will denote by $Y_{L}\left(t_{4}\right)$ (see Figs. 1 $\& 2$ ), and the rate of change in the $Y$-direction, $\dot{Y}_{L}$. Winn et al. (2006) choose $t_{4}$ and $t_{5}$ because of its tighter connections with observations $\left(t_{4}, t_{5}\right.$ denote changes in the light curve of $\left.\mathrm{KH} 15 \mathrm{D}\right)$. When extending the Winn et al. (2006) model, we will also primarily refer to orbital contact times to parameterize the advance of the screen across the orbit of the binary (Fig. 2), but also frequently refer to $Y_{L}$ and $\dot{Y}_{L}$ as well.

\subsection{Our model for the KH 15D System}

Our photometric model builds off of Winn et al. (2006), and seeks to fit the light curve of $\mathrm{KH} 15 \mathrm{D}$ from 1955-2018 with minimal modifications (smallest number of additional parameters to describe the trailing edge, in relation to the leading edge). After 2012, the 

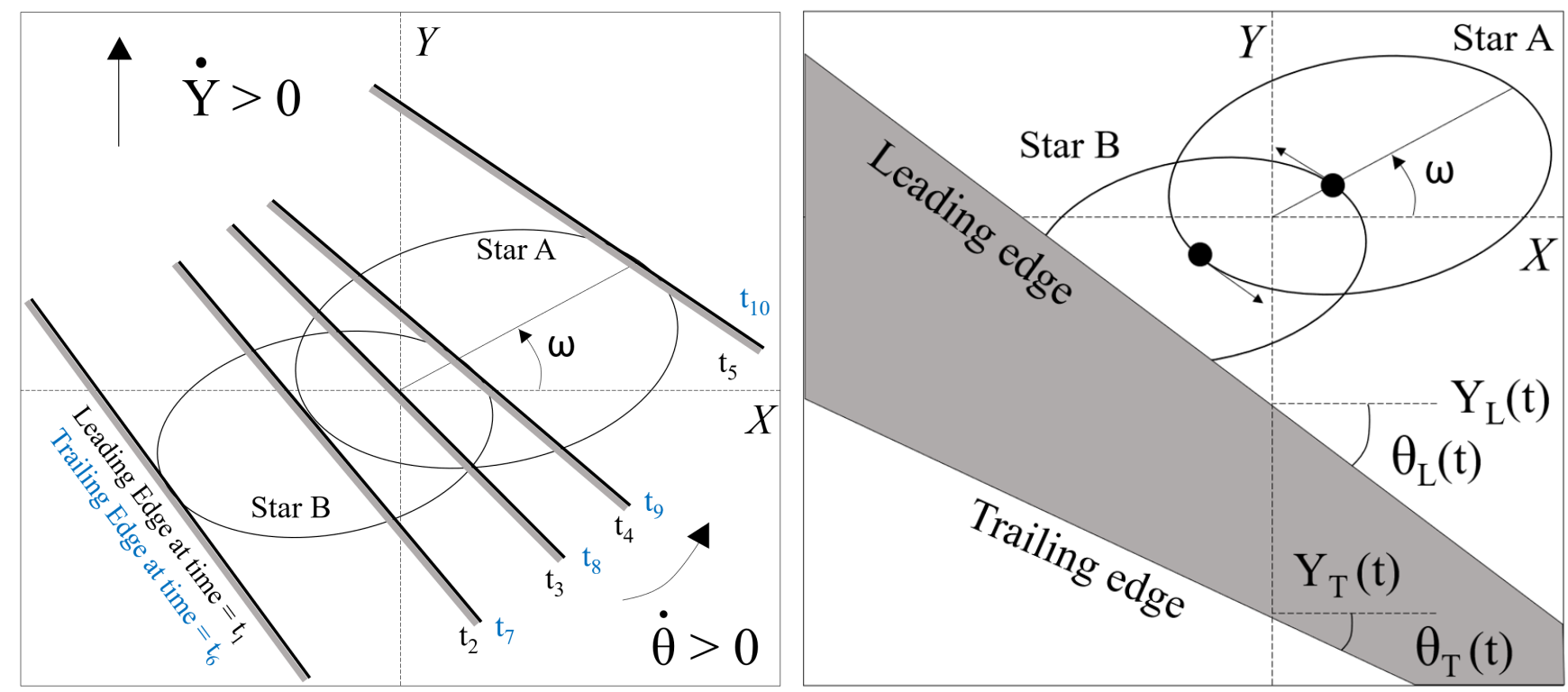

Figure 2. Left Panel: Definitions of orbital contact times. The leading (trailing) edge has contact times $t_{1}$ to $t_{5}\left(t_{6}\right.$ to $\left.t_{10}\right)$. All contact times denote when the leading or trailing edge lies tangent to the orbit of either star A or B, with the exception of $t_{3}$ and $t_{6}$, which denote when the leading or trailing edges intersect the center of mass of the binary. Right Panel: The definitions of quantities related to our model of the disk occulting the binary of KH 15D, which we model as an opaque screen bounded by two infinitely-long, straight edges on both sides. The leading (trailing) edge is parameterized by its intersection with the $Y$-axis, $Y_{L}\left(Y_{T}\right)$, and the angle between the edge and the $X$-axis, $\theta_{L}\left(\theta_{T}\right)$. Our model allows for $Y_{L}, \theta_{L}, Y_{T}$, and $\theta_{T}$ to evolve (linearly) with time. We emphasize our model makes no assumption on the underlying geometry of the disk occulting the binary of KH 15D.

trailing edge started to uncover star B, due to the other (inner or outer) truncation radius of the disk of $\mathrm{KH} 15 \mathrm{D}$ precessing over the binary orbit with respect to the line-of-sight of the observer (see Fig. 1). The simplest extension is to include an additional trailing edge in the modelling (denoted by subscript $T$ ), which lags in position behind the leading edge (with subscript $L$ ), which intersects the $Y$-axis at a location $Y_{T}(t)$ (see Fig. 2). This trailing edge also introduces 5 new orbital contact times as the edge crosses the orbit of the binary: $t_{6}, t_{7}, t_{8}, t_{9}, t_{10}$ (see Fig. 2 for illustration). Assuming $\theta_{L}=\theta_{T}$ and $\dot{Y}_{L}=\dot{Y}_{T}$, the previous 1-edge semi-infinite sheet becomes a 2-edge thin rectangular sheet of constant width, which is infinite along its length. García Soto et al. (2020) assumed this for their light curve model, and neatly fit CCD photometry from 1995 and onwards. However, because this fit does not match the light curve data prior to 1995 (fit not shown here or in García Soto et al. 2020), further modifications are needed to the Winn et al. (2006) and García Soto et al. (2020) model.

Through much experimentation, we found the following set of additions to the Winn et al. (2006) model that let us fit the 60+ year light curve. The connection of these additions with a warped disk driven into precession by an eccentric binary will be made clear in the following section.

- We let the leading and trailing edges have different angles $\left(\theta_{L}[t] \neq \theta_{T}[t]\right.$, see Fig. 2).

- We let each edge linearly evolve in time independently $\left(\dot{\theta}_{L}[t] \neq\right.$ $\left.\dot{\theta}_{T}[t]\right)$.

- Parameterize $\dot{\theta}_{L}$ by two constant, piecewise rates in time: $\dot{\theta}_{L}(t)=\dot{\theta}_{L 1}$ when $t<t_{3}$, and $\dot{\theta}_{L}(t)=\dot{\theta}_{L 2}$ when $t>t_{3}$. We keep $\dot{\theta}_{T}(t)=$ constant as a single parameter. Because we make the leading edge symmetric about $t_{3}$, we fit for the times $\left\{t_{3}, t_{5}\right\}$ in our MCMC model to constrain $Y_{L}\left(t_{3}\right)$ and $\dot{Y}_{L}$, rather than $\left\{t_{4}, t_{5}\right\}$ as in Winn et al. (2006).
Table 1. Definitions of Model Parameters.

\begin{tabular}{ll}
\hline \hline Free Parameter & Description \\
\hline$P$ & Orbital period \\
$e$ & Orbital eccentricity \\
$I$ & Inclination of orbital plane \\
$\omega$ & Argument of pericenter \\
$T_{p}$ & Time of periapsis passage \\
$L_{B} / L_{A}$ & Luminosity of star B relative to star A \\
$\epsilon_{1}$ & Fractional flux of stellar halo \\
$\epsilon_{2}$ & Fractional flux of stellar halo \\
$\xi_{1}$ & Exponential scale factor of stellar halo 1 \\
$\xi_{2}$ & Exponential scale factor of stellar halo \\
$t_{3}$ & Third orbital contact time \\
$t_{5}$ & Fifth orbital contact time \\
$t_{6}$ & Sixth orbital contact time \\
$\theta_{L}\left(t_{3}\right)$ & Angle between x-axis and leading edge at $t=t_{3}$ \\
$\theta_{T}\left(t_{3}\right)$ & Angle between x-axis and trailing edge at $t=t_{3}$ \\
$\dot{\theta}_{L 1}$ & Rotation rate of leading edge when $t<t_{3}$ \\
$\dot{\theta}_{L 2}$ & Rotation rate of leading edge when $t>t_{3}$ \\
$\dot{\theta}_{T}$ & Rotation rate of trailing edge \\
\hline
\end{tabular}

${ }^{1}$ In the direction the leading edge approaches the star

${ }^{2}$ In the direction the leading edge travels beyond the star

${ }^{3}$ Defined in Figure 2

- Let the width of the screen change over time $\left(\dot{Y}_{L} \neq \dot{Y}_{T}\right)$, but keep both rates $\dot{Y}_{L}$ and $\dot{Y}_{T}$ constant with time.

- Prescribe the rate of ascent of the trailing edge in relation to the rate of ascent of the leading edge. Specifically, we take $\dot{Y}_{T}=\alpha \dot{Y}_{L}$ for $\alpha=0.1,0.3,0.5,2.0,3.0,10.0$. We also experimented with letting $\dot{Y}_{T}$ be a free parameter (fitting for the contact times $\left\{t_{6}, t_{7}\right\}$ ), and found these fits gave $\dot{Y}_{T} \approx \dot{Y}_{L}$, but the MCMC did not always converge. We 
choose this parameterization to make sure the other model parameters are well-determined.

We further simplify the Winn model by analytically solving for $L_{A}$ and $\gamma$ with respect to the rest of the parameters, since they are constant shifts to the photometric and radial velocity models, respectively. This reduces the number of free parameters by 2 . For reference, we display each model parameter and its definition in Table 1.

To calculate the flux from the KH 15D system, we simply add the flux from stellar light emitted exterior to the trailing edge, to that emitted exterior to the leading edge. In more detail, letting $y_{T, i}$ be the distance of the trailing edge from star $i$, with $v_{T, i}=y_{T, i} / R_{i}$, star $i$ emits the flux

$F_{T, i}=\int_{-\infty}^{v_{T, i}} B_{i}(v) \mathrm{d} v$

exterior to the trailing edge, giving the total flux $F=F_{L, \mathrm{~A}}+F_{L, \mathrm{~B}}+$ $F_{T, \mathrm{~A}}+F_{T, \mathrm{~B}}$. We neglect the intersection between the leading and trailing edges in the flux calculation, because this intersection occurs far from the orbit of the binary.

For our radial velocity model, we follow equations (2) and (3) from Section 2.1 of Fulton et al. (2018). To optimize the model parameters, we use a Python-implemented Markov chain Monte Carlo (MCMC) package emcee by Foreman-Mackey et al. (2013). We use the same $\chi^{2}$ statistic as in equation (4).

Preliminary tests find the background light in the KH 15D system to be $L_{0} \approx 0$, so we remove $L_{0}$ from our model parameters. This is expected if $L_{0}$ is from forward scattering of the stars' light around the trailing edge of the disk (Silvia \& Agol 2008), rather than the finite optical depth of the disk itself (Chiang \& Murray-Clay 2004), because forward scattering of stellar light around both screen edges is included in our model. Our final model has 18 parameters (see Table 1), which we run for 20,000 steps with 36 walkers. Running the final model for each $\alpha$, we come to the following results: each MCMC converged except for $\alpha=10.0$, with only $\alpha=0.3,0.5$ producing reasonable-looking light curves. Model parameters for $\alpha=0.1,0.3,2.0,3.0$ are reported in Appendix A1. We highlight $\alpha=0.5$ as the best fit with parameters in Table 2, and display corner plots of the posteriors in Appendix B1. Because no stellar eclipses have been detected in the KH 15D light curve (only the disk-binary occultations), we fix the binary inclination to be $I \geq 91^{\circ}$ in our MCMC analysis, so the system is not perfectly edge-on.

Our fit for the entire light curve of KH 15D is displayed in Figure 3. Our model does a good job in describing both the maximum and minimum fluxes from $\mathrm{KH} 15 \mathrm{D}$, which change with time. As expected, the orbital contact times $t_{i}$ denote when the light curve of the system changes its morphology. The gradual change of maximum/minimum flux around $t_{i}$ values is due to the halos around each star: for point-source stars occulted by a razor-thin opaque edge, the photometric model predicts almost discontinuous changes in light curve morphologies around $t_{i}$ values.
Table 2. Model fits to photometric and radial velocity data for the KH $15 \mathrm{D}$ system, taking $\alpha=0.5$. Orbital parameters $\left\{P, e, I, \omega, T_{p}\right\}$ are constrained using photometry and radial velocity data, while the other parameters are constrained using photometry alone.

\begin{tabular}{|c|c|}
\hline Parameter & Our Fit \\
\hline$P$ [days $]$ & $48.3777_{-0.0002}^{+0.0002}$ \\
\hline$e$ & $0.5784_{-0.0009}^{+0.0009}$ \\
\hline$I[\mathrm{deg}]$ & $91.001_{-0.001}^{+0.002}$ \\
\hline$\omega[\operatorname{deg}]$ & $11.80_{-0.06}^{+0.06}$ \\
\hline$T_{p}[\mathrm{JD}]-2,452,350$ & $4.18_{-0.02}^{+0.01}$ \\
\hline$L_{B} / L_{A}$ & $1.65_{-0.01}^{+0.01}$ \\
\hline$\epsilon_{1}$ & $0.0436_{-0.0006}^{+0.0006}$ \\
\hline$\epsilon_{2}$ & $\begin{array}{l}0.0591_{-0.0008}^{+0.0008} \\
0.08\end{array}$ \\
\hline$\xi_{1}$ & $1.53_{-0.03}^{+0.03}$ \\
\hline$\xi_{2}$ & $2.86_{-0.03}^{+0.03}$ \\
\hline$t_{3}$ & $1992.68_{-0.05}^{+0.05}$ \\
\hline$t_{5}$ & $2007.95_{-0.01}^{+0.01}$ \\
\hline$t_{6}$ & $2013.57_{-0.03}^{+0.03}$ \\
\hline$\theta_{L}\left(t_{3}\right)[\mathrm{deg}]$ & $-16.0_{-0.2}^{+0.03}$ \\
\hline$\theta_{T}\left(t_{3}\right)[\mathrm{deg}]$ & $-5.3_{-0.2}^{+0.2}$ \\
\hline$\dot{\theta}_{L 1}[\mathrm{rad} /$ year $]$ & $0.0077_{-0.0002}^{+0.0002}$ \\
\hline$\dot{\theta}_{L 2}[\mathrm{rad} /$ year $]$ & $0.0033_{-0.0001}^{+0.0001}$ \\
\hline$\dot{\theta}_{T}[\mathrm{rad} / \mathrm{year}]$ & $-0.0006_{-0.0002}^{+0.0001}$ \\
\hline$\chi_{\text {phot }}^{2}$ & 13325 \\
\hline$\chi_{\mathrm{RV}}^{2}$ & 13 \\
\hline Reduced $\chi^{2}$ & 1.36 \\
\hline$t_{1}$ & $1972.9 \pm 0.2^{1}$ \\
\hline$t_{2}$ & $1987.00 \pm 0.01^{1}$ \\
\hline$t_{4}$ & $1996.8 \pm 0.1^{1}$ \\
\hline$t_{7}$ & $2020.8 \pm 0.1^{1}$ \\
\hline$t_{8}$ & $2024.95 \pm 0.01^{1}$ \\
\hline$t_{9}$ & $2028.6 \pm 0.1^{1}$ \\
\hline$t_{10}$ & $2041.0 \pm 0.5^{1}$ \\
\hline$Y_{T}\left(t_{3}\right)[\mathrm{au}]$ & $-0.05903^{2}$ \\
\hline$Y_{L}\left(t_{6}\right)[\mathrm{au}]$ & $0.07642^{2}$ \\
\hline$Y_{T}\left(t_{6}\right)[\mathrm{au}]$ & $-0.02082^{2}$ \\
\hline$\dot{Y}_{L}\left(t_{6}\right)[\mathrm{au} / \mathrm{year}]$ & $0.003658^{2}$ \\
\hline$\dot{Y}_{T}\left(t_{6}\right)[\mathrm{au} /$ year $]$ & $0.001829^{2}$ \\
\hline
\end{tabular}

Figures 4-5 show the photometric model and data folded over the binary orbital period, which is comparable to Figure 12 in Winn et al. (2006). Again, we see our model does a good job at modelling changes in the light curve of KH $15 \mathrm{D}$, with orbital contact times (see Table 2 for values) delineating morphology changes as one or both stars becomes occulted or revealed by an edge. Examining the data from 2013-2014 and 2015-2016, the large scatter makes it seem unlikely that any (simple) model could provide an accurate fit to the observed light curve. In addition, we do not remove any outliers (compare 1998-1999 panel in Fig. 4 to Fig. 12 in Winn et al. 2006). An interesting feature occurs around 2010, when the egress is poorly fit (for all values of $\alpha$ ). This could be related to the clumpiness/transparency near the edges of the disk as discussed in García Soto et al. (2020), where the assumption of sharp edges breaks down. 


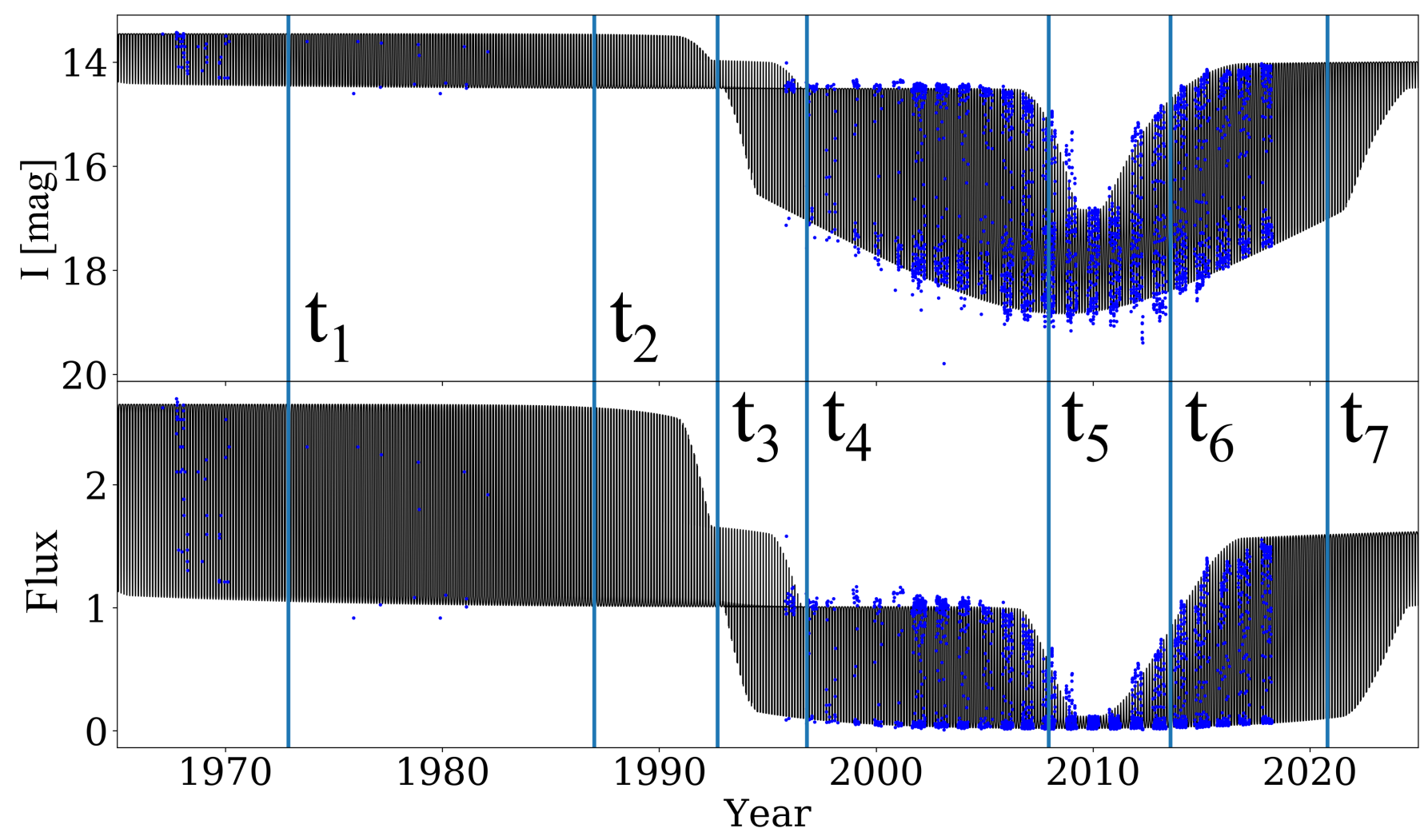

Figure 3. Light curve of KH 15D from 1965 to present, displaying the complex change in variability seen with time. The observed light curve in $I$-band magnitude is shown in the upper panel, and the light curve after the normalization to the flux of star A is shown in the lower panel. Blue points are photometry from Aronow et al. (2018) and García Soto et al. (2020), while the thin black line displays our photometric model fit (see Table 2 for parameter values). Vertical cyan lines denote the orbital contact times $t_{i}$ indicated, where the leading or trailing edge of the screen (e.g. circumbinary disk, see Fig. 1) hits a different portion of the binary orbit (see Fig. 2 for definitions). Our model does well in reproducing the KH 15D light curve variability over the length of time the system is observed.

Table 3. An excerpt from Table 3. For interested observers, the full version (available in machine-readable form) includes $I$ band magnitude predictions for the years 2000-2050 from our light curve model of KH 15D as shown in Figure 3, Figure 4 and Figure 5.

\begin{tabular}{ccc}
\hline Julian Date & Gregorian Date & KH 15D I-band magnitude \\
\hline 2451545.0 & 2000.000 & 14.509 \\
2451546.0 & 2000.003 & 14.507 \\
2451547.0 & 2000.005 & 14.506 \\
$\ldots$ & $\ldots$ & $\ldots$ \\
2469807.0 & 2049.999 & 13.462 \\
\hline
\end{tabular}

\section{A DYNAMICAL MODEL FOR THE DISK OF KH 15D}

The previous section showed that in order for the Winn et al. (2006) model to fit the entire more than 60 year light curve of KH $15 \mathrm{D}$, a number of modifications to this original model must be made. In this section, we illustrate how these modifications are motivated by the dynamics of a warped disk, driven into precession around an eccentric binary. In doing so, we will show that the disk orientation, warp, radial extent, and even surface density profile may be constrained by photometry alone.

\subsection{Model for a Precessing, Warped Circumbinary Disk Orbiting KH 15D}

For the disk around KH 15D to coherently precess over its lifetime, internal forces within the disk must keep neighboring disk annuli nearly aligned with one another, otherwise differential nodal precession from the gravitational influence of the binary will disrupt and "break" the disk (e.g. Larwood \& Papaloizou 1997; Facchini et al. 2013; Nixon et al. 2013; Martin \& Lubow 2018). When these internal torques are much stronger than the external torque on the disk from the binary, the disk behaves as a rigid body, coherently precessing about the orbital angular momentum axis of the binary (e.g. Martin \& Lubow 2017; Smallwood et al. 2019; Moody et al. 2019). To model the dynamical evolution of the disk, we will assume the disk behaves approximately like a rigid plate, treating the disk as a secondary whose mass is distributed between radii $r_{L}$ and $r_{T}$.

However, before we introduce our model for an extended disk, we discuss the dynamics of a test particle on a circular orbit (which we will refer to as a ring), driven into precession by the torque from the binary. Many authors have shown the orbital angular momentum unit vector of the ring $\hat{l}_{\mathrm{r}}$ is driven into precession and nutation about either the orbital angular momentum unit vector of the binary $\hat{l}$, or eccentricity vector of the binary $\boldsymbol{e}$ (vector in pericenter direction with magnitude $e$ ). The dynamical evolution of the ring depends sensitively on the initial orientation of $\hat{l}_{\mathrm{r}}$ with respect to $\hat{\boldsymbol{l}}$ and $\boldsymbol{e}$, as well as the magnitude of the eccentricity of the binary $e$. To calculate 


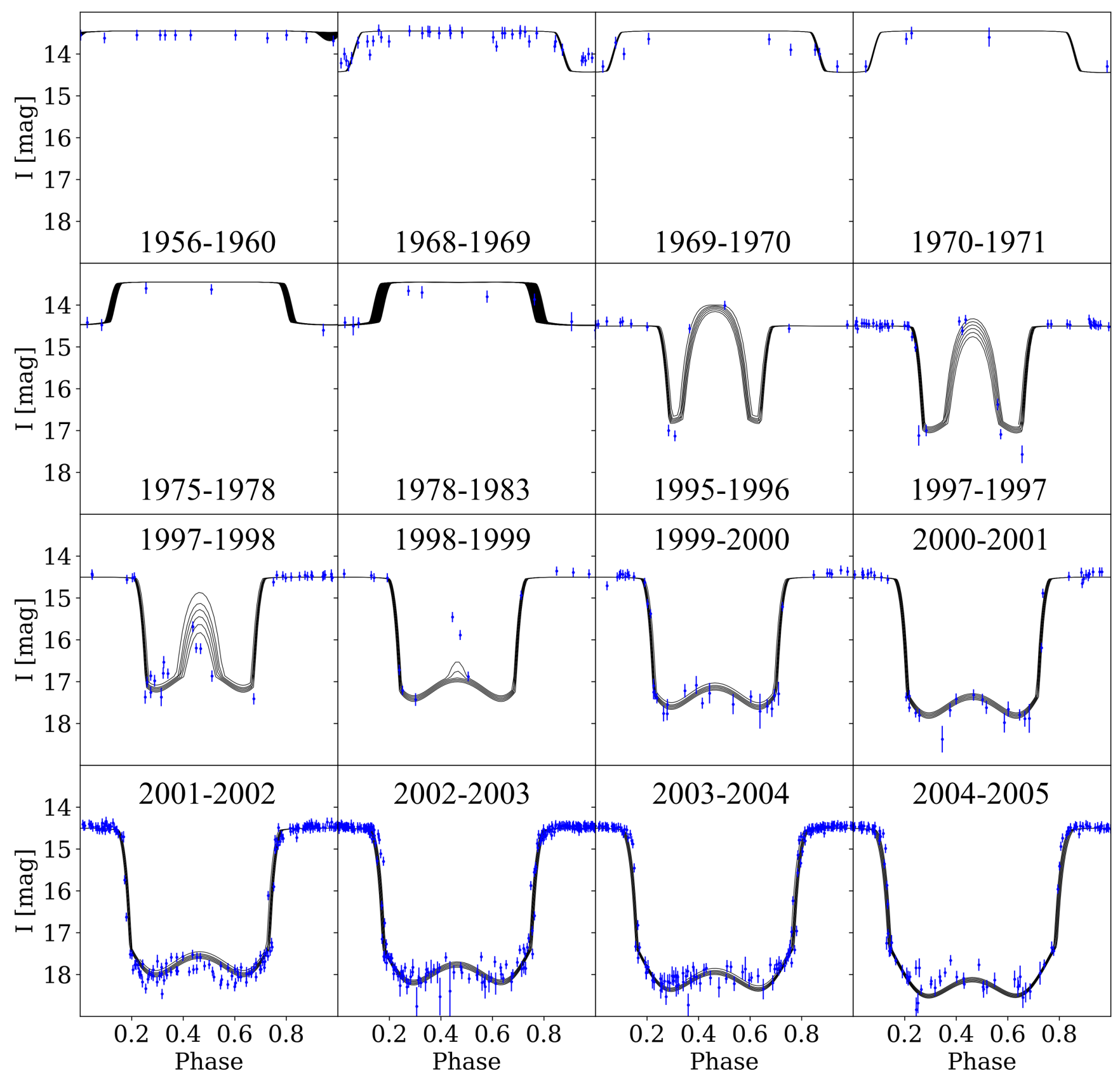

Figure 4. Data (blue points) and fitted model (black lines) displayed in Figure 3, folded over the binary orbital period, prior to the year 2005. The timespan over which the data and model are folded over is displayed in each figure. Our model reproduces the changing morphology of the light curve of KH $15 \mathrm{D}$ well.

the evolution of $\hat{\boldsymbol{l}}_{\mathrm{r}}$ about $\hat{\boldsymbol{l}}$ and $\boldsymbol{e}$, we adopt the formalism of Farago \& Laskar (2010), who calculated the secular evolution of a ring about a massive binary with an eccentric orbit, after expanding the Hamiltonian of the binary to leading order in $r / a$ (where $r$ is the semimajor axis of the test particle), and averaging over the mean motions of the test particle and the binary. It was found the characteristic precession and nutation frequency of $\hat{l}_{\mathrm{r}}$ about the binary was given by (denoted by $\alpha$ in Farago \& Laskar 2010)

$v=\frac{3 \mu}{4 M_{\mathrm{t}}}\left(\frac{G M_{\mathrm{t}}}{a^{3}}\right)^{1 / 2}\left(\frac{a}{r}\right)^{7 / 2}$, where $M_{\mathrm{t}}=M_{\mathrm{A}}+M_{\mathrm{B}}$ is the total mass of the binary, while $\mu=$ $M_{\mathrm{A}} M_{\mathrm{B}} / M_{\mathrm{t}}$ is the reduced mass of the binary.

After calculating the evolution of a (circular) test particle $\hat{l}_{\mathrm{r}}$ vector about $\hat{\boldsymbol{l}}$ and $\boldsymbol{e}$ using Farago \& Laskar (2010), we then translate the evolution of $\hat{l}_{\mathrm{r}}$ into the inclination of the test particle $I_{\mathrm{r}}$ and longitude of ascending node $\Omega_{\mathrm{r}}$, in the frame where $\hat{z}=\hat{\boldsymbol{l}}$ and the line of nodes points in the direction of $\boldsymbol{e}$. Because the orientation of the binary orbit in the reference frame of a distant observer is described by the orbital elements $\{a, e, \omega, I, \Omega\}$, the position of the ring in the frame 


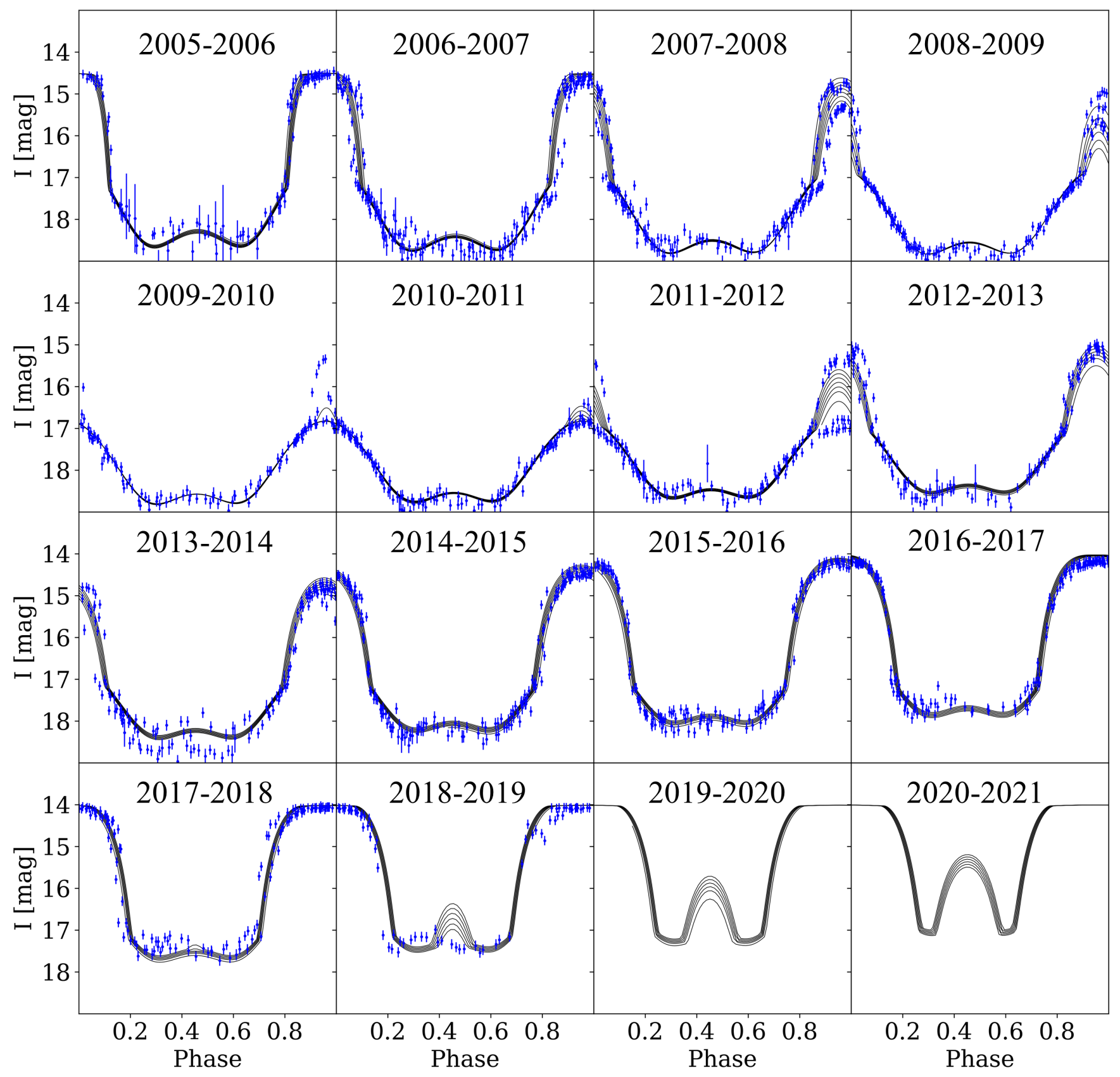

Figure 5. Same as Figure 4, except for data and model fits after the year 2005. We also display model predictions for the years 2019 to 2021.

of the observer is

$\left(\begin{array}{c}x \\ y \\ z\end{array}\right)_{\mathrm{r}, \mathrm{obs}}=R_{Z}(\Omega) R_{X}(I) R_{Z}(\omega) R_{Z}\left(\Omega_{\mathrm{r}}\right) R_{X}\left(I_{\mathrm{r}}\right)\left(\begin{array}{c}x_{\mathrm{r}} \\ y_{\mathrm{r}} \\ 0\end{array}\right)$,

where $\left(x_{\mathrm{r}}, y_{\mathrm{r}}\right)=r(\cos \varphi, \sin \varphi)$ parameterizes the $(X, Y)$ coordinates of the ring in the frame where $\hat{z}=\hat{\boldsymbol{l}}$, and $R_{X}[\beta]\left(R_{Z}[\gamma]\right)$ denote rotations along the $X(Z)$-axis by angles $\beta(\gamma)$. As in Winn et al. (2006), we choose the reference plane of the observer so the $X$-axis points along the binary line-of-nodes (so $\Omega=0$ ). Also, because our MCMC model highly favors a nearly edge-on orbit (Table 2), we assume $I \simeq 90^{\circ}$ for simplicity for the rest of this section. All other orbital parameters are taken as their most likely values from Table 2.
To connect with a model for a disk occulting the binary of $\mathrm{KH}$ $15 \mathrm{D}$, we approximate the inner and outer edges of the disk as two rings with different orbital elements $\left\{r_{k}, I_{k}, \Omega_{k}\right\}$, with $k=L, T$ for the leading and trailing edges of the disk, respectively. Although each ring has a different $r_{k}$, we assume the rings precess about $\hat{l}$ with the same global disk precession frequency $v_{\mathrm{d}}$.

To connect the geometry of a disk occulting the binary of KH 15D with the edges of the light curve model in Section 2, we approximate an occulting ring by a line drawn tangent to the ring at the location where the ring intersects the $X$-axis of the system (see Fig. 6). The angle between the tangent line and $X$-axis $\theta_{k}$, as well as the $Y$ - 


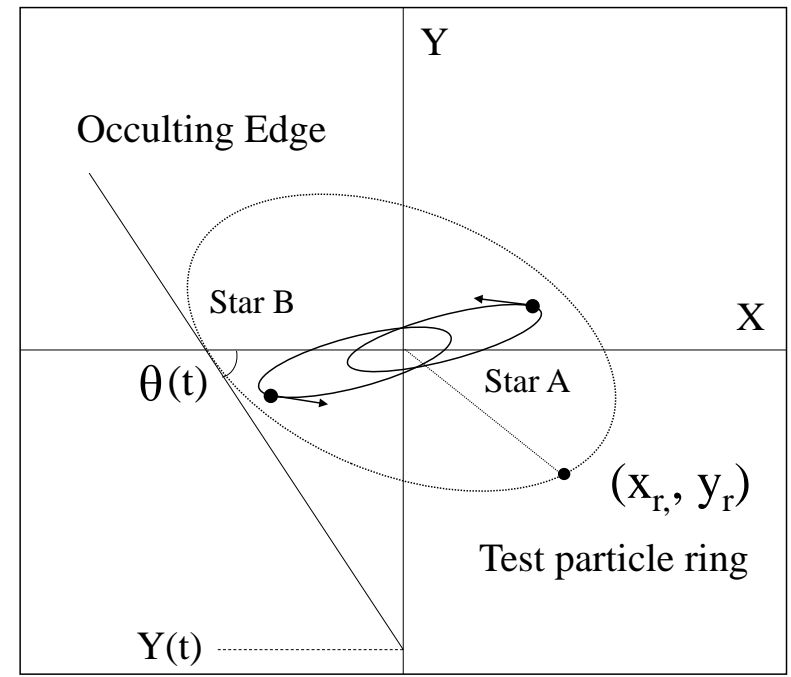

Figure 6. Our interpretation for the leading/trailing edges of the opaque screen in our photometry model. The leading/trailing edges of the screen are from the inner or outer disk truncation radii. The occulting disk edge is approximated by a straight line, drawn tangent to the intersection of the ring with the $X$-axis of the coordinate system. The $\theta=\theta_{k}$ and $Y=Y_{k}$ values of the leading/trailing edge are defined similarly as in Figure 2. Because the binary is nearly edge-on, the straight-line approximation is excellent.

intercept $Y_{k}$, of ring $k$ are then given by

$$
\begin{aligned}
& \theta_{k}\left[I_{k}(t), \Omega_{k}(t)\right]=\tan ^{-1}\left[\frac{\tan I_{k}}{\sin \left(\omega+\Omega_{k}\right)}\right], \\
& Y_{k}\left[I_{k}(t), \Omega_{k}(t)\right]=-\frac{r_{k} \tan I_{k}}{\tan \left(\omega+\Omega_{k}\right)} .
\end{aligned}
$$

A successful model of the circumbinary disk of KH 15D would give values for $\theta_{k}$ and $Y_{k}$ which match the MCMC fits for $\theta_{L}, \theta_{T}, Y_{L}$, and $Y_{T}$, from Table 2 .

\subsection{Estimates of Disk Properties from Model Fits}

Before presenting an example warped disk geometry which matches the light curve model fit, we discuss how the warped disk geometry can be constrained by the MCMC fits of Section 2.3. To do this, we simplify equations (8)-(9), and derive order-of-magnitude estimates for all disk quantities. Because the pericenter direction of the binary is nearly perpendicular to the observer $(\omega \ll 1)$, the disk annuli longitude of ascending nodes satisfy $\Omega_{k} \approx \pi / 2$ during transit. The disk inclination is also nearly aligned with the orbital plane of the binary $\left(\left|I_{k}\right| \ll 1\right)$. Also, because the binary pericenter direction is nearly perpendicular to the observer, the inclination nutations should be near a local minimum (e.g. Farago \& Laskar 2010; Zanazzi \& Lai 2018), so $\dot{I}_{k} \approx 0$. The nodal regression rate of the rings should be of order $\dot{\Omega}_{k} \approx-v_{k}$, where $v_{k}$ is the nearly constant nodal precession rate of ring $k$. Defining $\delta \Omega_{k} \equiv \Omega_{k}-\pi / 2$, and assuming $|\omega|,\left|I_{k}\right|$, and $\left|\delta \Omega_{k}\right| \ll 1$, equations (8)-(9) can be shown to reduce to

$\theta_{k} \approx I_{k}$

$\dot{\theta}_{k} \approx-\left(\omega+\delta \Omega_{k}\right) I_{k} v_{k}$,

$Y_{k} \approx r_{k} I_{k}\left(\omega+\delta \Omega_{k}\right)$,

$\dot{Y}_{k} \approx-r_{k} I_{k} v_{k}$.
From this, we see the increase of $Y_{L}$ and $Y_{T}$ is primarily due to nodal regression from the rings. The evolution of $\theta_{L}$ and $\theta_{T}$ is primarily due to the curvature of the ring, as it nodally precesses in front of the orbit of the binary (see Silvia \& Agol 2008 for further discussion). Most interestingly, the MCMC constraints on $\theta_{L}$ and $\theta_{T}$ directly translate to constraints on the ring inclinations $I_{L}$ and $I_{T}$.

Assuming the disk precesses rigidly $\left(v_{L} \approx v_{T}\right)$, one can then constrain the disk radial extent. Equation (13) leads to

$\frac{r_{L}}{r_{T}} \approx \frac{\theta_{T}}{\theta_{L}} \frac{\dot{Y}_{L}}{\dot{Y}_{T}}=0.63\left(\frac{\theta_{T}}{-5^{\circ}}\right)\left(\frac{-16^{\circ}}{\theta_{L}}\right)\left(\frac{0.5}{\alpha}\right)$.

Because the values of $\alpha$ which fit the data are of order unity $(0.3 \lesssim$ $\alpha \lesssim 1$ ), we can be confident that the leading edge of the screen occulting the binary of $\mathrm{KH} 15 \mathrm{D}$ is the disk inner truncation radius, while the trailing edge is the outer truncation radius $\left(r_{L} \lesssim r_{T}\right)$.

Moreover, because $\dot{\theta}_{k}, Y_{k}$, and $\dot{Y}_{k}$ are all known, one can get unique solutions for $r_{k}, \delta \Omega_{k}$, and $v_{k}$. Starting with $v_{k}$, equations (11)-(13) can be re-arranged to give

$v_{k} \approx\left(\frac{\dot{\theta}_{k} \dot{Y}_{k}}{Y_{k}}\right)^{1 / 2}$.

Evaluating estimate (15) at $t=t_{4}$, we find $v_{L} \sim 0.013 \mathrm{yr}^{-1}$ and $v_{T} \sim 0.0073 \mathrm{yr}^{-1}$, which are consistent with one another within a factor of a few. Similarly, equation (13) can be solved for $r_{k}$ :

$r_{k} \approx-\frac{1}{\theta_{k}}\left(\frac{Y_{k} \dot{Y}_{k}}{\dot{\theta}_{k}}\right)^{1 / 2}$,

which gives $r_{L} \sim 1.0 \mathrm{au}$ and $r_{T} \sim 4.6 \mathrm{au}$ at $t=t_{4}$ for our model. Last, either equation (11) or (12) can be solved for $\delta \Omega_{k}$.

Although these disk parameter estimates are far from unique, they provide constraints on the properties of the circumbinary disk within the KH $15 \mathrm{D}$ system. We can strongly conclude the disk-binary mutual inclination $I_{\mathrm{KH} 15 \mathrm{D}}$ in the $\mathrm{KH} 15 \mathrm{D}$ system lies in the range $5^{\circ} \lesssim$ $I_{\mathrm{KH} 15 \mathrm{D}} \lesssim 16^{\circ}$, with the disk inner edge more highly inclined than the outer edge (because $I_{L} \gtrsim I_{T}$ ). The leading edge of the opaque screen crossing the binary orbit is from the disk inner edge, which is located at a radius $r_{L} \lesssim 1$ au, while the trailing outer disk edge is located at $r_{T} \sim$ few au.

\subsection{Example Warped Disk which Matches Model Fits}

As we saw in the previous section, for a unique match to the phenomenological parameters $\left\{\theta_{k}, \dot{\theta}_{k}, Y_{k}, \dot{Y}_{k}\right\}$ to a precessing, inclined ring annulus, we require the ring parameters $\left\{r_{k}, I_{k}, \Omega_{k}, v_{k}\right\}$. However, for a protoplanetary disk to exist over many dynamical times, it must precess rigidly $\left(v_{L}=v_{T}\right)$, decreasing the number of free parameters in one ring. Therefore, our dynamical model is over-determined by our phenomenological model. To get accurate constraints on the warped disk itself using photometry, a light curve model must be developed whose free parameters are directly related to the warped disk properties (disk inclination, warp, twist, precession frequency, etc.), rather than indirectly through a phenomenological model. The goal of this section is not to provide stringent constraints on the disk itself, but to present an example warped disk which gives gross light curve features consistent with the MCMC light curve fits.

Motivated by the estimated leading and trailing estimates in the previous subsection, we experiment with the warped disk orbital parameters and global precession frequency, to find a disk whose properties match the MCMC fitted parameters. Table 4 presents example model parameters for a dynamically evolving, warped disk whose features are compatible with the light curve fits, with Figure 7 


\begin{tabular}{lr}
\hline Parameter & Example Value \\
\hline$v_{\mathrm{d}}\left[\mathrm{yr}^{-1}\right]$ & 0.01 \\
$r_{L}[\mathrm{AU}]$ & 0.5 \\
$r_{T}[\mathrm{AU}]$ & 2.0 \\
$I_{L}\left(t_{3}\right)[\mathrm{deg}]$ & -13 \\
$I_{T}\left(t_{3}\right)[\mathrm{deg}]$ & -6 \\
$\Omega_{L}\left(t_{3}\right)[\mathrm{deg}]$ & 100 \\
$\Omega_{T}\left(t_{3}\right)[\mathrm{deg}]$ & 115 \\
\hline
\end{tabular}

Table 4. Parameter values for our dynamical model of a warped disk precessing around an eccentric binary in the KH 15D system. Disk inclinations are relative to the binary orbital plane, and disk longitude of ascending nodes are relative to the binary pericenter direction. See text for definitions and discussion.
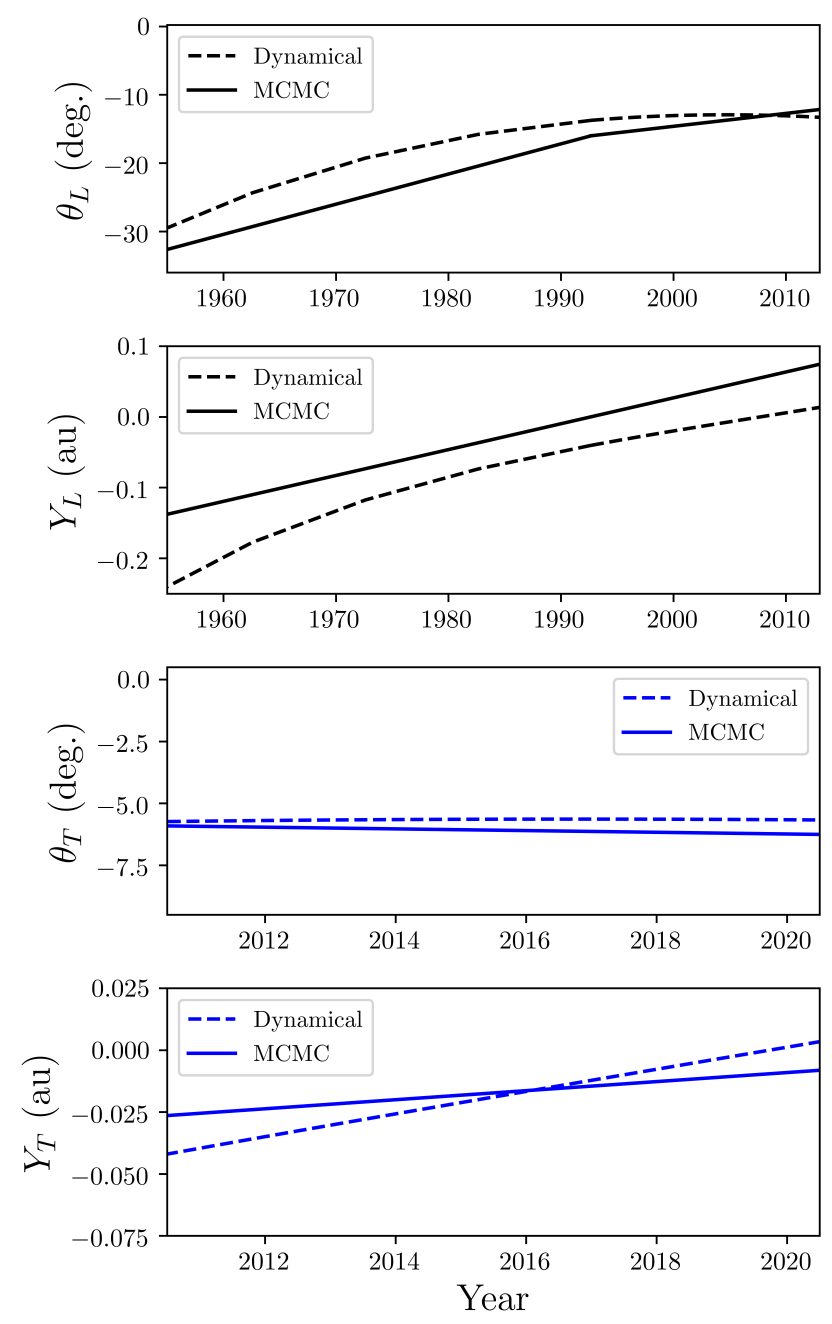

Figure 7. Comparing our dynamical warped disk model (Table 4) with the MCMC fits from our phenomenological photometry model (Table 2). Although agreement between the two models can be improved, the warped disk reproduces the main features of the photometry model.

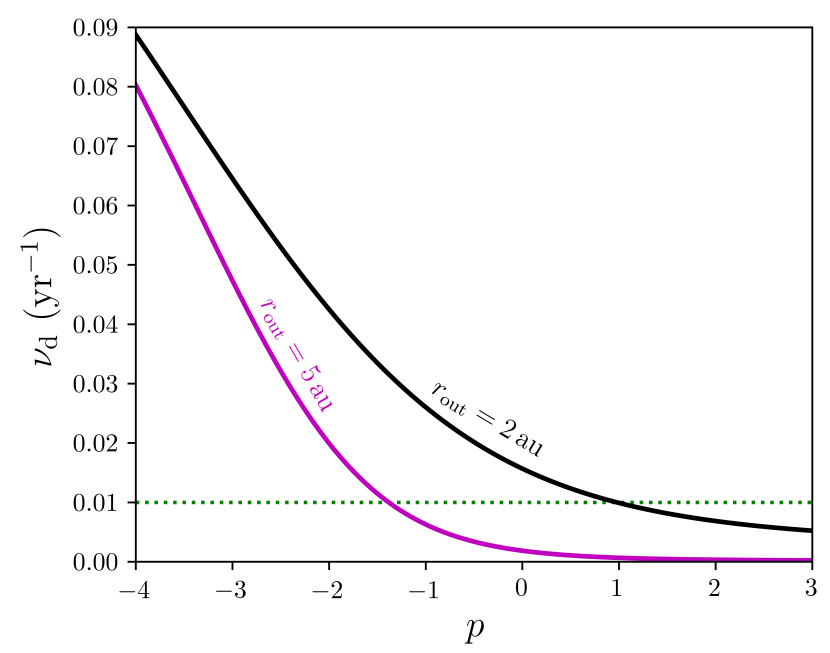

Figure 8. Global disk precession frequnecy $v_{\mathrm{d}}$ (eq. 17) as a function of the surface density powerlaw index $p\left(\Sigma \propto r^{p}\right)$, for the KH 15D system parameters, assuming $r_{\text {in }}=0.5$ au with the $r_{\text {out }}$ values indicated. Dotted green line shows our dynamical model value of $v_{\mathrm{d}}=0.01 \mathrm{yr}^{-1}$. Depending on the disk radial extent, a measurement of $v_{\mathrm{d}}$ translates to a constraint on $p$.

displaying $\theta_{k}(t)$ and $Y_{k}(t)$ for both (dynamical and MCMC) models over the duration of time the leading and trailing occultations have been observed. The dynamical model and MCMC fits match one another within a factor of a few, heavily reinforcing the idea that the light curve of $\mathrm{KH} 15 \mathrm{D}$ is caused by a warped, relatively narrow, precessing disk, occulting the starlight of the eccentric binary. In particular, we see the behavior of the dynamical model matches the $\theta_{L}(t)$ light curve fit, reproducing the decrease in $\dot{\theta}_{L}$ before and after the year $t=t_{3} \simeq 1993$.

Complimentary constraints on the disk of KH 15D have come recently from the double-peaked line profile of neutral oxygen emission, assuming the $[\mathrm{OI}] \lambda 6300$ emission originates from the surface of the gaseous circumbinary disk of KH 15D (Fang et al. 2019). This line profile was used to constrain the disk radial extent, as well as the disk surface density profile. Fang et al. (2019) found an inner disk radius of $r_{\text {in }} \approx 0.57$ au, an outer radius $r_{\text {out }} \approx 5.2$ au, and surface density profile $\Sigma \propto r^{-2.9}$. The inner and outer radii are roughly consistent with our $r_{L}=r_{\text {in }}$ and $r_{T}=r_{\text {out }}$ values constrained by our crude dynamical fit to the photometry of KH 15D (Table 4). We note that the outer edge of a protoplanetary disk gas and dust radius may differ, due to radial drift of the dust (e.g. Weidenschilling 1977; Takeuchi \& Lin 2002; Birnstiel \& Andrews 2014; Powell et al. 2017; Rosotti et al. 2019). Indeed, molecular line and continuum emission have been shown to extend to different radii around young stellar objects (e.g. Panić et al. 2009; Andrews et al. 2012; de GregorioMonsalvo et al. 2013; Ansdell et al. 2018; Facchini et al. 2019), showing gas and dust in protoplanetary disks often extend out to different radii (sometimes differing by as much as a factor of $\sim 3$ ). The dynamics of dust in a precessing circumbinary disk can also be non-trivial (Poblete et al. 2019; Aly \& Lodato 2020).

Our warped disk model can also constrain the disk surface density profile $\Sigma \propto r^{p}$, because the distribution of mass within the disk affects the torque exerted on the disk by the binary, modifying the disk precession frequency $v_{\mathrm{d}}$. Assuming a nearly-flat disk which is driven into rigid-body precession about the binary, $v_{\mathrm{d}}$ can be shown to be (e.g. Lodato \& Facchini 2013; Foucart \& Lai 2014; Zanazzi \& 
Lai 2018; Lubow \& Martin 2018)

$v_{\mathrm{d}}=\frac{3}{4}\left(\frac{5 / 2+p}{1-p}\right)\left[\frac{1-\left(r_{\mathrm{out}} / r_{\mathrm{in}}\right)^{p-1}}{\left(r_{\mathrm{out}} / r_{\mathrm{in}}\right)^{5 / 2+p}-1}\right] \frac{\mu}{M_{\mathrm{t}}}\left(\frac{a}{r_{\mathrm{in}}}\right)^{2} \sqrt{\frac{G M_{\mathrm{t}}}{r_{\text {in }}^{3}}}$.

Figure 8 plots the $v_{\mathrm{d}}$ value given by equation (17) as a function of $p$. Depending on the disk outer radius, we clearly see a measurement of $v_{\mathrm{d}}$ can constrain the $p$ value of the disk. The model parameters from Table 4 support $p \sim 1$, which differs substantially from the Fang et al. (2019) constraint of $p \approx-2.9$. However, we note that this discrepancy relies on the disk [OI] emission arising from a gaseous disk associated with the occulting ring, as opposed to the interpretation given by Mundt et al. (2010), who argued the [OI] emission originated from a bipolar jet associated with one or both of the stars at the center of KH 15D. Further photometric modelling is required to see if the $p$ value implied by the disk precession frequency differs from that constrained by the disk OI emission.

\section{THEORETICAL IMPLICATIONS OF DYNAMICAL WARPED DISK MODEL}

In $\S 3$, we showed how the photometry of KH $15 \mathrm{D}$ could be explained by a precessing circumbinary disk, in agreement with the results of other works (Winn et al. 2004, 2006; Chiang \& Murray-Clay 2004; Silvia \& Agol 2008). The parameters constrained by the photometry of the system are listed in Table 4. Although the fit of the dynamical model to the photometry is crude, we argue the basic conclusions on the parameters of the system are unlikely to differ by more than a factor of a few, and comprises some of the first constraints on small warps within protoplanetary disks. This section connects the constraints of our dynamical model to theories describing warp propagation in accretion disks, as well as speculation on the long-term evolution of the system. We also discuss predictions from our model, as well as future modelling efforts.

\subsection{Explaining the Warp and Twist within KH 15D}

Our dynamical model requires a non-zero warp $\left(\Delta I=I_{T}-I_{L}\right)$ and twist $\left(\Delta \Omega=\Omega_{T}-\Omega_{L}\right)$ to cause the complex series of occultations seen in the KH $15 \mathrm{D}$ system. These warps and twists arise from the disk resisting the differential nodal precession induced by the specific torque of the binary

$\left|\boldsymbol{T}_{\text {bin }}\right| \sim r^{2} n v|\bar{I}|$,

where $n=\sqrt{G M_{\mathrm{t}} / r^{3}}$ is the rings orbital frequency, $v$ is the characteristic nodal precession frequency induced on the disk from the binary (eq. 6), and $\bar{I}$ is the characteristic "average" inclination of the disk. One way to balance the torque from the binary is by thermal pressure between ringlets, which has an internal torque of order (e.g. Ogilvie 1999; Chiang \& Culter 2003; Chiang \& Murray-Clay 2004)

$\left|\boldsymbol{T}_{\text {press }}\right| \sim c_{\mathrm{s}}^{2}|\Delta I|$,

where $c_{\mathrm{S}}=h r n$ is the ring sound-speed, while $h$ is the aspect ratio of the disk. Assuming torque balance $\left(\left|\boldsymbol{T}_{\text {press }}\right| \approx\left|\boldsymbol{T}_{\text {bin }}\right|\right)$ allows us to estimate the warp which may develop under the resisting influence of thermal pressure:

$$
\begin{aligned}
& \left.\left|\frac{\Delta I}{\bar{I}}\right|_{\text {press }} \sim \frac{r^{2} n v}{c_{\mathrm{S}}^{2}}\right|_{r=\bar{r}} \\
& =13\left(\frac{0.05}{h}\right)^{2}\left(\frac{\mu}{0.33 \mathrm{M}_{\odot}}\right)\left(\frac{1.32 \mathrm{M}_{\odot}}{M_{\mathrm{t}}}\right)\left(\frac{a}{0.29 \mathrm{au}}\right)^{2}\left(\frac{0.7 \mathrm{au}}{\bar{r}}\right)^{2},
\end{aligned}
$$

where $\bar{r}$ is some characteristic radius within the disk. Clearly, this warp is quite large.

However, in nearly-inviscid (Shakura-Sunyaev parameter $\alpha \lesssim h$ ) disks with the radial-epicyclic frequency satisfying $\kappa^{2} \approx n^{2}$, the nearresonant propagation of bending waves across the disk can amplify the strength of the hydrodynamical torque by a factor (Papaloizou \& Lin 1995; Lubow \& Ogilvie 2000; Ogilvie 2006)

$\left|\boldsymbol{T}_{\mathrm{bw}}\right| \sim \frac{1}{|\tilde{\kappa}|}\left|\boldsymbol{T}_{\text {press }}\right|$,

where $\tilde{\kappa} \equiv\left(\kappa^{2}-n^{2}\right) /\left(2 n^{2}\right)$ is a dimensionless quantity related to the apsidal precession rate. Because the secular apsidal precession rate is $|\tilde{\kappa}| \sim v / n$ for circumbinary disks (Miranda \& Lai 2015), torque balance $\left(\left|\boldsymbol{T}_{\mathrm{bw}}\right| \approx\left|\boldsymbol{T}_{\text {bin }}\right|\right)$ gives

$$
\begin{aligned}
& \left.\left|\frac{\Delta I}{\bar{I}}\right|_{\mathrm{bw}} \sim \frac{r^{2} v^{2}}{c_{\mathrm{S}}^{2}}\right|_{r=\bar{r}} \\
& =0.42\left(\frac{0.05}{h}\right)^{2}\left(\frac{\mu}{0.33 \mathrm{M}_{\odot}}\right)^{2}\left(\frac{1.32 \mathrm{M}_{\odot}}{M_{\mathrm{t}}}\right)^{2}\left(\frac{a}{0.29 \mathrm{au}}\right)^{4}\left(\frac{0.7 \mathrm{au}}{\bar{r}}\right)^{4} .
\end{aligned}
$$

This estimate is much closer to the $|\Delta I / \bar{I}| \sim 1$ values implied by our dynamical model, and lies in agreement with more detailed calculations of warp propagation in protoplanetary disks (Lodato \& Facchini 2013; Foucart \& Lai 2013, 2014; Zanazzi \& Lai 2018; Lubow \& Martin 2018).

Disk self-gravity can also resist differential nodal precession from the binary. Mutually-misaligned ringlets experience specific mutual internal torques of order (Chiang \& Culter 2003; Chiang \& MurrayClay 2004; Tremaine \& Davis 2014; Zanazzi \& Lai 2017; Batygin 2018)

$\left|\boldsymbol{T}_{\mathrm{sg}}\right| \sim \frac{G \Sigma r}{h}|\Delta I| \sim \frac{G M_{\mathrm{d}}}{h r}|\Delta I|$,

assuming the disk mass $M_{\mathrm{d}} \sim r^{2} \Sigma$, and the additional factor of $h^{-1}$ arises from the enhancement of the mutual gravitational attraction between ringlets when the disk is vertically thin (Batygin 2018). Torque balance $\left(\left|\boldsymbol{T}_{\mathrm{sg}}\right| \approx\left|\boldsymbol{T}_{\text {bin }}\right|\right)$ leads to warps of order

$$
\begin{aligned}
& \left.\left|\frac{\Delta I}{\bar{I}}\right|_{\mathrm{sg}} \sim \frac{r^{3} n v h}{G M_{\mathrm{d}}}\right|_{r=\bar{r}} \\
& =1.3\left(\frac{h}{0.05}\right)\left(\frac{\mu}{0.33 \mathrm{M}_{\odot}}\right)\left(\frac{1.7 \mathrm{M}_{\mathrm{Jup}}}{M_{\mathrm{d}}}\right)\left(\frac{a}{0.29 \mathrm{au}}\right)^{2}\left(\frac{0.7 \mathrm{au}}{\bar{r}}\right)^{2} .
\end{aligned}
$$

Even after assuming the upper limit on the total (gas and dust) disk mass inferred by ALMA observations (Aronow et al. 2018), self-gravity is typically not as effective as bending waves at enforcing coplanarity between ringlets. However, a massive disk $\left(M_{\mathrm{d}} \sim\right.$ $1 \mathrm{M}_{\mathrm{Jup}}$ ) can give warps comperable to those inferred by our dynamical KH 15D disk model.

The direction of the warp ( $\Delta I$ positive or negative) has also been argued to encode information on the internal forces/torques enforcing disk coplanarity. Chiang \& Murray-Clay (2004) argued thermal pressure predicts $\Delta I<0$, while self-gravity predicts $\Delta I>0$. More detailed calculations support the prediction that a disk should relax to a $\Delta I>0$ profile under the influence of disk self-gravity (Batygin 2012, 2018; Zanazzi \& Lai 2017). But calculations taking into account the resonant propagation of bending waves also predict $\Delta I>0$ (e.g. Facchini et al. 2013; Foucart \& Lai 2014; Zanazzi \& Lai 2018; Lubow \& Martin 2018). Hydrodynamical simulations of protoplanetary disks (neglecting self-gravity) find conflicting results, with $\Delta I>0$ and $\Delta I<0$ at different times, primarily because the 
simulations usually cannot be run long enough for the system to relax to a smoothly-evolving warp profile (e.g. Facchini et al. 2013; Martin \& Lubow 2017, 2018; Smallwood et al. 2019, 2020; Moody et al. 2019). We note that the disk may never relax to a steady-state. Simulations which accurately calculate how the binary interacts with a tidally-truncated circumbinary disk find highly-dynamical inner disk edges for disks orbiting eccentric binaries (e.g. Miranda et al. 2017; Muñoz et al. 2019, 2020; Franchini et al. 2019). Because resonant Lindblad torques often truncate disks (e.g. Artymowicz \& Lubow 1994; Lubow et al. 2015; Miranda \& Lai 2015), which may also excite disk tilts (Borderies et al. 1984; Lubow 1992; Zhang \& Lai 2006), it is not unreasonable to say a real circumbinary disk may never relax to a steady-state inclination profile. We conclude that bending-wave propagation is the main internal force enforcing rigid precession of the disk of $\mathrm{KH} 15 \mathrm{D}$, despite the conflicting predictions for the sign of $\Delta I$.

A small viscosity in a circumbinary disk also leads to a nonzero twist, due to the azimuthal shear induced by differential nodal precession. The magnitude of the torque resisting nodal shear is (Papaloizou \& Pringle 1983; Papaloizou \& Lin 1995; Ogilvie 1999; Lubow \& Ogilvie 2000)

$\left|\boldsymbol{T}_{\mathrm{visc}}\right| \sim \frac{1}{\alpha} c_{\mathrm{s}}^{2}|\Delta \Omega|$,

assuming an isotropic kinematic viscosity $(v=\alpha p /[\rho n])$. The $\alpha^{-1}$ (rather than $\alpha^{+1}$ ) dependence in equation (25) is from near-resonant forcing of radial and azimuthal perturbations ( are damped only by viscosity (Papaloizou \& Lin 1995; Lubow \& Ogilvie 2000; Lodato \& Pringle 2007). Viscosity leads to twists of order (assuming $\left|\boldsymbol{T}_{\text {visc }}\right| \approx\left|\boldsymbol{T}_{\text {bin }}\right|$ )

$$
\begin{aligned}
&\left|\frac{\Delta \Omega}{\bar{I}}\right|_{\text {visc }}\left.\sim \frac{\alpha r^{2} n v}{c_{\mathrm{s}}^{2}}\right|_{r=\bar{r}} \\
&=0.13\left(\frac{\alpha}{0.01}\right)\left(\frac{0.05}{h}\right)^{2}\left(\frac{\mu}{0.33 \mathrm{M}_{\odot}}\right) \\
& \times\left(\frac{1.32 \mathrm{M}_{\odot}}{M_{\mathrm{t}}}\right)\left(\frac{a}{0.29 \mathrm{au}}\right)^{2}\left(\frac{0.7 \mathrm{au}}{\bar{r}}\right)^{2} .
\end{aligned}
$$

More detailed calculations typically give positive $\Delta \Omega$ values a bit larger in circumbinary disks (Foucart \& Lai 2014; Zanazzi \& Lai 2018), in agreement with our dynamical model. Although observations frequently infer $\alpha$ values much lower than $10^{-2}$ (e.g. Hughes et al. 2011; Flaherty et al. 2015; Teague et al. 2016; Rafikov 2017; Ansdell et al. 2018), the large warp in this disk can excite parametric instabilities, enhancing the viscous dissipation rate in the disk (Goodman 1993; Ryu \& Goodman 1994; Gammie et al. 2000; Ogilvie \& Latter 2013; Paardekooper \& Ogilvie 2019).

The arguments above slightly favor a disk held together by resonant bending waves over self-gravity. However, such an interpretation requires the scaleheight of the gas be much higher than that of the solids (dust, pebbles, or planetesimals), which must be sufficiently small to cause the sharp occultations seen in the KH 15D lightcurve $\left(H_{\text {solid }} \lesssim R_{\mathrm{A}}, R_{\mathrm{B}}\right)$. Although no firm detection of disk gas within the KH 15D system has been made, Lawler et al. (2010) detected Na I D line emission and absorption from KH $15 \mathrm{D}$, with a column density which did not vary as the stars became more inclined to the disk midplane. If the $\mathrm{Na}$ I D emission/absorbtion is from the disk gas (not the interstellar medium), this implies a large gas scaleheight $\left(H_{\text {gas }} \gg R_{\mathrm{A}}, R_{\mathrm{B}}\right)$. A discrepancy between the gas and solid scaleheights is expected theoretically, as aerodynamical drag causes particles to settle to the disk midplane (e.g. Youdin \& Lithwick 2007). Without gas, dust/solids/planetesimals tend to have larger

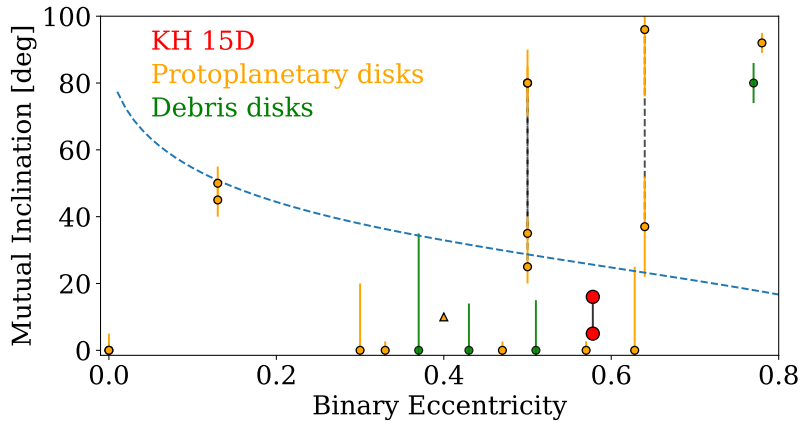

Figure 9. The mutual inclination between the disk and binary orbital plane in the KH 15D system (red), plotted alongside circumbinary disk inclinations for protoplanetary (orange) and debris (green) disks (Czekala et al. 2019), as a function of binary eccentricity. The dashed blue lines plots the critical inclination (eq. 27). The black dotted lines connect degenerate solutions for HD 142527, SR 24N and GG Tau Aa-Ab. The triangle represents the lower limit for R CrA. The disk in KH 15D will align (not polar align) with the orbital plane of the binary.

scaleheights due to mutual gravitational interactions which excite particle inclinations (e.g. Goldreich et al. 2004). Moreover, if the disk has no gas, because the solids must be optically-thick to starlight, the required solid densities would cause frequent collisions between particles, and imply the KH 15D disk has a short lifetime (e.g. Wyatt 2008).

We conclude the disk warp and twist implied by our model lie in accord with hydrodynamical theories of warped accretion disks.

\subsection{Long-Term Dynamical Evolution of KH 15D}

Recently, Czekala et al. (2019) showed circumbinary disks (both gas and debris) have higher inclinations when orbiting eccentric binaries. Figure 9 displays the disk inclinations analyzed in Czekala et al. (2019), alongside our constraints for the inclination of $\mathrm{KH}$ $15 \mathrm{D}$, which we take directly from our photometric fits $\left(\left|\theta_{T}\left[t_{3}\right]\right| \lesssim\right.$ $\left|I_{\mathrm{KH} 15 \mathrm{D}}\right| \lesssim\left|\theta_{L}\left[t_{3}\right]\right|$, see $\left.\S 3.2\right)$. The dashed blue line displays the critical inclination (Aly et al. 2015; Martin \& Lubow 2017; Zanazzi \& Lai 2018)

$I_{\text {crit }}=\cos ^{-1} \sqrt{\frac{5 e^{2}}{1+4 e^{2}}}$,

which is a necessary (but not sufficient) condition for the disk-binary inclination to evolve to $90^{\circ}$ (polar alignment). From Figure 9, because $\left|I_{\text {crit }}\right|>\left|I_{\mathrm{KH} 15 \mathrm{D}}\right|$, we can be confident the disk will not polar align, and will eventually align with the orbital plane of the binary (without any other mechanisms exciting the disk inclination).

We can also estimate the timescale over which the disk inclination evolves. Because a non-zero twist $\Delta \Omega$ exerts a backreaction torque on the disk from the binary, the disk is driven into alignment (or polar alignment) over the timescale (Foucart \& Lai 2014; Zanazzi \& Lai 2018)

$\gamma_{\mathrm{evol}} \sim v_{\mathrm{d}}|\Delta \Omega|$.

Inserting equation (26) into equation (28) gives the often-quoted "Bate timescale" (Bate et al. 2000). However, because $v_{\mathrm{d}}$ and $\Delta \Omega$ are both determined by our dynamical model, we can actually estimate 
$\gamma_{\mathrm{evol}}$ using observationally inferred parameters:

$\gamma_{\mathrm{evol}} \sim 2.6 \times 10^{-3}\left(\frac{v_{\mathrm{d}}}{0.01 \mathrm{yr}^{-1}}\right)\left(\frac{\Delta \Omega}{15^{\circ}}\right) \mathrm{yr}^{-1}$.

Equation (29) implies the disk should align with the orbital plane of the binary in less than $\sim 10^{3}$ years. Although secular interactions can keep the disk misaligned with the eccentric orbital plane of the binary over timescales a few times longer than estimate (29) (Zanazzi \& Lai 2018; Smallwood et al. 2019), this is much shorter than the $\sim 10^{6}$ year lifetimes of typical protoplanetary disks (e.g. Haisch et al. 2001). Either we are observing KH 15D while it is still very young, or additional mechanisms are exciting the disk inclination.

\subsection{KH 15D Model Predictions and Improvements}

The most immediate consequences are predictions for future light curve behavior from our photometry model fits (Figs. $3 \& 5$, Table 2). Current $I$-band measurements should show the light from star A slowly being revealed by the trailing edge (since $t_{7} \approx 2021$ ). By the year $\sim 2029$, the orbit of star B should be completely revealed, resulting in a ceasing of the variability from this star. By the year $\sim 2041$, we should cease to see photometric variability due to the circumbinary disk. While our current model which produces a reasonable fit to the photometric data employs an opaque screen with a constant $\dot{\theta}_{T}$, our dynamical model predicts that the fit can be further improved if the change of $\dot{\theta}_{T}$ with time is incorporated (eq. 11). We provide in Table 3 the predicted light curve (in $I$ band) of this system until the year 2050 .

We are able to make an explicit connection between the phenomenological model $(\$ 2)$, and a precessing, warped disk occulting the binary of $\mathrm{KH} 15 \mathrm{D}(\S 3)$. More stringent constraints on the disk geometry would use the inner and outer disk orbital parameters $\left\{r_{k}, I_{k}, \Omega_{k}\right\}$ and global disk precession frequency $v_{\mathrm{d}}$, rather than parameters describing the locations and orientations of the leading and trailing edges $\left\{\theta_{k}, \dot{\theta}_{k}, Y_{k}, \dot{Y}_{k}\right\}$, to fit the light curve of KH 15D. This exercise should yield parameters consistent with those listed in Table 4 within a factor of a few.

Our folded light curves (Figs 4-5) show the leading edge is well fit by a sharp edge, whereas the poor fit for the trailing edge imply it is clumpy/puffy, in agreement with the findings of García Soto et al. (2020). The sharp inner edge is likely due to tidal truncation by the torque from the binary. Calculations and hydrodynamical simulations suggest that the radius at which the binary truncates the disk is $\sim 2$ times the binary semi-major axis $(a \approx 0.3 \mathrm{au})$ (e.g. Miranda \& Lai $2015)$, lying close to the inner radius value of our dynamical model (Table 4). However, it remains unclear why the disk is so compact ( $r_{\text {out }} \lesssim 5 \mathrm{au}$ ), and the possibility still exists the disk outer edge is truncated by a planet (Chiang \& Murray-Clay 2004). There exists tentative observational support for this hypothesis, as Arulanantham et al. (2017) found infrared-excess from KH 15D consistent with the thermal emission from a $\sim 10 M_{\text {Jup }}$ mass planet. Future modelling of how dust scattering and the finite optical depth of the disk can create a "fuzzier" outer edge would be of interest (Chiang \& Murray-Clay 2004; Silvia \& Agol 2008).

\section{CONCLUSIONS}

In this work, we have developed a circumbinary disk model that explains the photometric variability of KH $15 \mathrm{D}$ spanning more than 60 years. From this model, we are able to constrain the disk annular extent, inclination, orientation with respect to the binary pericenter direction, warp profile, precession frequency, and even surface density profile. The fits of our phenomenological model to fit the photometry of $\mathrm{KH} 15 \mathrm{D}$ are displayed in Table 2, with parameters of a warped disk which are consistent with the phenomenological model constraints listed in Table 4. Although strict constraints on the warped disk remain elusive, we can be confident about the following features of the disk:

- The beginning of the dips/occultations in $\mathrm{KH} 15 \mathrm{D}$ are due to the disk inner edge slowly covering the binary, while the currently observed slow reversal of the dipping behavior in $\mathrm{KH} 15 \mathrm{D}$ is due to the disk outer edge slowly revealing the binary. The inner edge has a radius $r_{\text {in }} \lesssim 1$ au, while the outer edge has a radius $r_{\text {out }} \sim$ few au.

- The disk inner edge is more inclined to the orbital plane of the binary than the disk outer edge. Both inner and outer disk inclinations are less than $\sim 16^{\circ}$, but greater than $\sim 5^{\circ}$, with a difference of order $\sim 10^{\circ}$.

- The disk inner and outer longitude of ascending nodes differ by $\sim 15^{\circ}$.

These constraints are consistent with hydrodynamical theories of warped accretion disks, resisting differential nodal precession from the gravitational torque from the binary $(\$ 4.1)$.

Our models also find a precessional period of order $P_{\text {prec }} \sim$ $2 \pi / v_{\mathrm{d}} \sim 600$ years, but this constraint is sensitive to the model fit of KH $15 \mathrm{D}$. We can be very confident, however, that the timescale over which the disk of KH 15D aligns with the orbital plane of the binary is much shorter than the lifetime of the disk (eq. 29), suggesting that additional mechanisms are exciting the disk tilt.

\section{ACKNOWLEDGEMENTS}

We thank Eugene Chiang, Ruth Murray-Clay, and Norm Murray for useful discussions, and Jessica Speedie for help in developing the circumbinary disk visualization in Figure 1. We would also like to thank the referee, William Herbst, for comments and suggestions that have improved the quality of this work. MP has been supported by undergraduate research fellowships from the Centre for Planetary Sciences and Canadian Institute for Theoretical Astrophysics (CITA). $\mathrm{JZ}$ and $\mathrm{WZ}$ were supported by the Natural Sciences and Engineering Research Council of Canada (NSERC) under the funding reference \# CITA 490888-16.

\section{DATA AVAILABILITY}

The data underlying this article are available in the public domain (Hamilton et al. 2003; Johnson et al. 2004; Johnson \& Winn 2004; Maffei et al. 2005; Winn et al. 2006; Aronow et al. 2018; García Soto et al. 2020). The data produced in this work are available in its online supplementary material (Table 3).

\section{REFERENCES}

Aly H., Lodato G., 2020, MNRAS, 492, 3306

Aly H., Dehnen W., Nixon C., King A., 2015, MNRAS, 449, 65

Andrews S. M., Czekala I., Wilner D. J., Espaillat C., Dullemond C. P., Hughes A. M., 2010, ApJ, 710, 462

Andrews S. M., et al., 2012, ApJ, 744, 162

Ansdell M., et al., 2018, ApJ, 859, 21 
Armstrong D. J., Osborn H. P., Brown D. J. A., Faedi F., Gómez Maqueo Chew Y., Martin D. V., Pollacco D., Udry S., 2014, MNRAS, 444, 1873

Aronow R. A., Herbst W., Hughes A. M., Wilner D. J., Winn J. N., 2018, AJ, 155,47

Artymowicz P., Lubow S. H., 1994, ApJ, 421, 651

Arulanantham N. A., Herbst W., Gilmore M. S., Cauley P. W., Leggett S. K., 2017, ApJ, 834, 119

Bate M. R., 2012, MNRAS, 419, 3115

Bate M. R., 2018, MNRAS, 475, 5618

Bate M. R., Bonnell I. A., Clarke C. J., Lubow S. H., Ogilvie G. I., Pringle J. E., Tout C. A., 2000, MNRAS, 317, 773

Bate M. R., Bonnell I. A., Bromm V., 2002, MNRAS, 336, 705

Batygin K., 2012, Nature, 491, 418

Batygin K., 2018, MNRAS, 475, 5070

Bi J., et al., 2020, ApJ, 895, L18

Birnstiel T., Andrews S. M., 2014, ApJ, 780, 153

Borderies N., Goldreich P., Tremaine S., 1984, ApJ, 284, 429

Brinch C., Jørgensen J. K., Hogerheijde M. R., Nelson R. P., Gressel O., 2016, ApJ, 830, L16

Capelo H. L., Herbst W., Leggett S. K., Hamilton C. M., Johnson J. A., 2012, ApJ, 757, L18

Chen C., Franchini A., Lubow S. H., Martin R. G., 2019, MNRAS, 490, 5634

Chen C., Lubow S. H., Martin R. G., 2020, MNRAS, 494, 4645

Chiang E. I., Culter C. J., 2003, ApJ, 599, 675

Chiang E. I., Murray-Clay R. A., 2004, ApJ, 607, 913

Czekala I., Andrews S. M., Jensen E. L. N., Stassun K. G., Torres G., Wilner D. J., 2015, ApJ, 806, 154

Czekala I., Andrews S. M., Torres G., Jensen E. L. N., Stassun K. G., Wilner D. J., Latham D. W., 2016, ApJ, 818, 156

Czekala I., et al., 2017, ApJ, 851, 132

Czekala I., Chiang E., Andrews S. M., Jensen E. L. N., Torres G., Wilner D. J., Stassun K. G., Macintosh B., 2019, ApJ, 883, 22

Doolin S., Blundell K. M., 2011, MNRAS, 418, 2656

Doyle L. R., Deeg H. J., 2018, The Way to Circumbinary Planets. p. 115, doi:10.1007/978-3-319-55333-7_115

Facchini S., Lodato G., Price D. J., 2013, MNRAS, 433, 2142

Facchini S., Juhász A., Lodato G., 2018, MNRAS, 473, 4459

Facchini S., et al., 2019, A\&A, 626, L2

Fang M., Pascucci I., Kim J. S., Edwards S., 2019, ApJ, 879, L10

Farago F., Laskar J., 2010, MNRAS, 401, 1189

Flaherty K. M., Hughes A. M., Rosenfeld K. A., Andrews S. M., Chiang E., Simon J. B., Kerzner S., Wilner D. J., 2015, ApJ, 813, 99

Foreman-Mackey D., Hogg D. W., Lang D., Goodman J., 2013, PASP, 125, 306

Foucart F., Lai D., 2013, ApJ, 764, 106

Foucart F., Lai D., 2014, MNRAS, 445, 1731

Franchini A., Lubow S. H., Martin R. G., 2019, ApJ, 880, L18

Francis L., van der Marel N., 2020, ApJ, 892, 111

Fulton B. J., Petigura E. A., Blunt S., Sinukoff E., 2018, PASP, 130, 044504

Gammie C. F., Goodman J., Ogilvie G. I., 2000, MNRAS, 318, 1005

García Soto A., Ali A., Newmark A., Herbst W., Windemuth D., Winn J. N., 2020, AJ, 159, 135

Giuppone C. A., Cuello N., 2019, in Journal of Physics Conference Series. p. 012023 (arXiv : 1907.08180), doi:10.1088/1742-6596/1365/1/012023

Goldreich P., Lithwick Y., Sari R., 2004, ARA\&A, 42, 549

Goodman J., 1993, ApJ, 406, 596

Haisch Karl E. J., Lada E. A., Lada C. J., 2001, ApJ, 553, L153

Hamilton C. M., Herbst W., Mundt R., Bailer-Jones C. A. L., Johns-Krull C. M., 2003, ApJ, 591, L45

Hamilton C. M., et al., 2005, AJ, 130, 1896

Hughes A. M., Wilner D. J., Andrews S. M., Qi C., Hogerheijde M. R., 2011, ApJ, 727, 85

Johnson J. A., Winn J. N., 2004, AJ, 127, 2344

Johnson J. A., Marcy G. W., Hamilton C. M., Herbst W., Johns-Krull C. M., 2004, AJ, 128, 1265

Kearns K. E., Herbst W., 1998, ApJ, 116, 261

Kennedy G. M., et al., 2012a, MNRAS, 421, 2264
Kennedy G. M., Wyatt M. C., Sibthorpe B., Phillips N. M., Matthews B. C., Greaves J. S., 2012b, MNRAS, 426, 2115

Kennedy G. M., et al., 2019, Nature Astronomy, 3, 230

Kraus S., et al., 2020, arXiv e-prints, p. arXiv:2004.01204

Larwood J. D., Papaloizou J. C. B., 1997, MNRAS, 285, 288

Lawler S. M., Herbst W., Redfield S., Hamilton C. M., Johns-Krull C. M., Winn J. N., Johnson J. A., Mundt R., 2010, ApJ, 711, 1297

Li G., Holman M. J., Tao M., 2016, ApJ, 831, 96

Lodato G., Facchini S., 2013, MNRAS, 433, 2157

Lodato G., Pringle J. E., 2007, MNRAS, 381, 1287

Lubow S. H., 1992, ApJ, 398, 525

Lubow S. H., Martin R. G., 2018, MNRAS, 473, 3733

Lubow S. H., Ogilvie G. I., 2000, ApJ, 538, 326

Lubow S. H., Martin R. G., Nixon C., 2015, ApJ, 800, 96

Maffei P., Ciprini S., Tosti G., 2005, MNRAS, 357, 1059

Marino S., Perez S., Casassus S., 2015, ApJ, 798, L44

Martin R. G., Lubow S. H., 2017, ApJ, 835, L28

Martin R. G., Lubow S. H., 2018, MNRAS, 479, 1297

Martin D. V., Triaud A. H. M. J., 2014, A\&A, 570, A91

McLaughlin D. B., 1924, ApJ, 60, 22

Miranda R., Lai D., 2015, MNRAS, 452, 2396

Miranda R., Muñoz D. J., Lai D., 2017, MNRAS, 466, 1170

Moody M. S. L., Shi J.-M., Stone J. M., 2019, ApJ, 875, 66

Muñoz D. J., Miranda R., Lai D., 2019, ApJ, 871, 84

Muñoz D. J., Lai D., Kratter K., Mirand a R., 2020, ApJ, 889, 114

Mundt R., Hamilton C. M., Herbst W., Johns-Krull C. M., Winn J. N., 2010, ApJ, 708, L5

Nixon C., King A., Price D., 2013, MNRAS, 434, 1946

Ogilvie G. I., 1999, MNRAS, 304, 557

Ogilvie G. I., 2006, MNRAS, 365, 977

Ogilvie G. I., Latter H. N., 2013, MNRAS, 433, 2420

Paardekooper S.-J., Ogilvie G. I., 2019, MNRAS, 483, 3738

Panić O., Hogerheijde M. R., Wilner D., Qi C., 2009, A\&A, 501, 269

Papaloizou J. C. B., Lin D. N. C., 1995, ApJ, 438, 841

Papaloizou J. C. B., Pringle J. E., 1983, MNRAS, 202, 1181

Pegues J., et al., 2021, arXiv e-prints, p. arXiv:2101.05838

Poblete P. P., Cuello N., Cuadra J., 2019, MNRAS, 489, 2204

Poblete P. P., Calcino J., Cuello N., Macías E., Ribas Á., Price D. J., Cuadra J., Pinte C., 2020, MNRAS, 496, 2362

Powell D., Murray-Clay R., Schlichting H. E., 2017, ApJ, 840, 93

Price D. J., et al., 2018, MNRAS, 477, 1270

Rafikov R. R., 2017, ApJ, 837, 163

Rosenfeld K. A., Andrews S. M., Wilner D. J., Stempels H. C., 2012, ApJ, 759,119

Rosotti G. P., Tazzari M., Booth R. A., Testi L., Lodato G., Clarke C., 2019, MNRAS, 486, 4829

Rossiter R. A., 1924, ApJ, 60, 15

Ruíz-Rodríguez D., Kastner J. H., Dong R., Principe D. A., Andrews S. M., Wilner D. J., 2019, AJ, 157, 237

Ryu D., Goodman J., 1994, ApJ, 422, 269

Silvia D. W., Agol E., 2008, ApJ, 681, 1377

Smallwood J. L., Lubow S. H., Franchini A., Martin R. G., 2019, MNRAS, 486, 2919

Smallwood J. L., Franchini A., Chen C., Becerril E., Lubow S. H., Yang C.-C., Martin R. G., 2020, MNRAS, 494, 487

Takeuchi T., Lin D. N. C., 2002, ApJ, 581, 1344

Teague R., et al., 2016, A\&A, 592, A49

Tremaine S., Davis S. W., 2014, MNRAS, 441, 1408

Weidenschilling S. J., 1977, MNRAS, 180, 57

Welsh W. F., Orosz J. A., 2018, Two Suns in the Sky: The Kepler Circumbinary Planets. p. 34, doi:10.1007/978-3-319-55333-7_34

Winn J. N., Holman M. J., Johnson J. A., Stanek K. Z., Garnavich P. M., 2004, ApJ, 603, L45

Winn J. N., Hamilton C. M., Herbst W. J., Hoffman J. L., Holman M. J., Johnson J. A., Kuchner M. J., 2006, ApJ, 644, 510

Wyatt M. C., 2008, ARA\&A, 46, 339

Youdin A. N., Lithwick Y., 2007, Icarus, 192, 588

Zanazzi J. J., Lai D., 2017, MNRAS, 464, 3945 
Zanazzi J. J., Lai D., 2018, MNRAS, 473, 603

Zhang Z., Fabrycky D. C., 2019, ApJ, 879, 92

Zhang H., Lai D., 2006, MNRAS, 368, 917

de Gregorio-Monsalvo I., et al., 2013, A\&A, 557, A133

\section{APPENDIX A: MODEL PARAMETERS}

We display all MCMC parameter fits for our new photometric model, for various $\alpha$, as described in Section 2.2. Recall $\alpha$ is the ratio of the trail edge velocity over the lead edge velocity along the vertical axis of our line of sight. Small $\alpha$ tests for narrow disk models whereas large $\alpha$ tests for extended disk models. We do not report model parameters for $\alpha=10.0$ since the MCMC does not converge. Upper and lower error bars indicate a $1 \sigma$ confidence interval. Model parameters are described in Table 1.

\section{APPENDIX B: MCMC CORNER PLOTS}

We display the corner plots to our best fit model $(\alpha=0.5)$ with best fit values listed in Table 2. We remove the first 17,500 of 20,000 total steps as burn-in, and plot the posterior distribution. The apparent degeneracy with $\xi_{2}$ and $L_{B}$ appears in many of the MCMC fits. This is likely due to some subtleties of the halo model, yet they do not affect the quality of the photometric fits. Because we are primarily interested in constraints on the properties describing the ascent of the leading and trailing screens $\left\{\theta_{k}, \dot{\theta}_{k}, Y_{k}, \dot{Y}_{k}\right\}$, to be consistent with Winn et al. (2006), we do not modify the halo model.

This paper has been typeset from a $\mathrm{T}_{\mathrm{E}} \mathrm{X} / \mathrm{L} \mathrm{T}_{\mathrm{E}} \mathrm{X}$ file prepared by the author. 
Table A1. Same as Table 2, except we vary the value of $\alpha$.

\begin{tabular}{|c|c|c|c|c|}
\hline Free parameter & $\alpha=0.1$ & $\alpha=0.3$ & $\alpha=2.0$ & $\alpha=3.0$ \\
\hline $\begin{array}{l}P \text { [days] } \\
e \\
I \text { [deg] } \\
\omega[\mathrm{deg}] \\
T_{p}[\mathrm{JD}]-2,452,350 \\
L_{B} / L_{A} \\
\epsilon_{1} \\
\epsilon_{2} \\
\xi_{1} \\
\xi_{2} \\
t_{3} \\
t_{5} \\
t_{6} \\
\theta_{L}\left(t_{3}\right)[\mathrm{deg}] \\
\theta_{T}\left(t_{3}\right) \text { [deg] } \\
\dot{\theta}_{L 1}[\mathrm{rad} / \text { year }] \\
\dot{\theta}_{L 2}[\mathrm{rad} / \text { year }] \\
\dot{\theta}_{T}[\mathrm{rad} / \text { year] }\end{array}$ & $\begin{array}{c}48.3786_{-0.0002}^{+0.0002} \\
0.5794_{-0.0008}^{+0.0008} \\
91.002_{-0.000}^{+0.003} \\
9.80_{-0.06}^{+0.05} \\
3.48_{-0.01}^{+0.02} \\
1.63_{-0.02}^{+0.01} \\
0.0516_{-0.0008}^{+0.0008} \\
0.097_{-0.001}^{+0.001} \\
1.81_{-0.04}^{+0.03} \\
7.5_{-0.2}^{+0.2} \\
1992.70_{-0.06}^{+0.06} \\
2008.16_{-0.01}^{+0.01} \\
2013.18_{-0.03}^{+0.03} \\
-55_{-1}^{+1} \\
-2.7_{-0.4}^{+0.4} \\
0.0075_{-0.0005}^{+0.0004} \\
0.0093_{-0.0003}^{+0.0003} \\
-0.0035_{-0.0003}^{+0.0003}\end{array}$ & $\begin{array}{c}48.3781_{-0.0002}^{+0.0002} \\
0.5771_{-0.0009}^{+0.0009} \\
91.002_{-0.000}^{+0.003} \\
11.29_{-0.06}^{+0.06} \\
4.00_{-0.02}^{+0.02} \\
1.57_{-0.01}^{+0.02} \\
0.0481_{-0.0007}^{+0.0007} \\
0.0651_{-0.0009}^{+0.0009} \\
1.44_{-0.02}^{+0.02} \\
3.60_{-0.06}^{+0.07} \\
1993.16_{-0.05}^{+0.05} \\
2008.12_{-0.01}^{+0.01} \\
2013.46_{-0.03}^{+0.03} \\
-20.0_{-0.3}^{+0.3} \\
-2.4_{-0.2}^{+0.2} \\
0.0099_{-0.0003}^{+0.0002} \\
0.0033_{-0.0002}^{+0.0002} \\
-0.0030_{-0.0001}^{+0.0001}\end{array}$ & $\begin{array}{c}48.3713_{-0.0002}^{+0.0002} \\
0.5716_{-0.00008}^{+0.0008} \\
91.0004_{-0.0000}^{+0.0008} \\
12.04_{-0.06}^{+0.06} \\
4.32_{-0.02}^{+0.02} \\
1.17_{-0.01}^{+0.01} \\
0.071_{-0.001}^{+0.001} \\
0.0495_{-0.0006}^{+0.0006} \\
2.40_{-0.04}^{+0.04} \\
2.97_{-0.05}^{+0.05} \\
1991.49_{-0.08}^{+0.00} \\
2007.72_{-0.01}^{+0.01} \\
2012.44_{-0.02}^{+0.02} \\
-13.5_{-0.2}^{+0.2} \\
-21.0_{-0.3}^{+0.3} \\
0.0025_{-0.0002}^{+0.0002} \\
0.0042_{-0.0001}^{+0.0001} \\
0.0065_{-0.0002}^{+0.0002}\end{array}$ & $\begin{array}{c}48.3691_{-0.0001}^{+0.0002} \\
0.5677_{-0.00008}^{+0.0008} \\
91.0005_{-0.00003}^{+0.0008} \\
11.97_{-0.07}^{+0.006} \\
4.31_{-0.02}^{+0.02} \\
1.064_{-0.009}^{+0.009} \\
0.085_{-0.001}^{+0.001} \\
0.0499_{-0.0007}^{+0.0007} \\
3.18_{-0.06}^{+0.07} \\
3.04_{-0.05}^{+0.05} \\
1991.3_{-0.1}^{+0.1} \\
2007.76_{-0.02}^{+0.02} \\
2012.07_{-0.02}^{+0.02} \\
-13.7_{-0.2}^{+0.2} \\
-29.7_{-0.5}^{+0.4} \\
0.0019_{-0.0003}^{+0.0003} \\
0.0046_{-0.0001}^{+0.0001} \\
0.0088_{-0.0003}^{+0.0003}\end{array}$ \\
\hline $\begin{array}{l}\text { Fit photometry? } \\
\chi_{\text {phot }}^{2} \\
\chi_{\mathrm{RV}}^{2} \\
\text { Reduced } \chi^{2}\end{array}$ & $\begin{array}{c}\text { No } \\
16386 \\
12 \\
1.62\end{array}$ & $\begin{array}{c}\text { Yes } \\
13822 \\
13 \\
1.40\end{array}$ & $\begin{array}{c}\text { No } \\
15558 \\
16 \\
1.60\end{array}$ & $\begin{array}{c}\text { No } \\
17222 \\
17 \\
1.76\end{array}$ \\
\hline
\end{tabular}




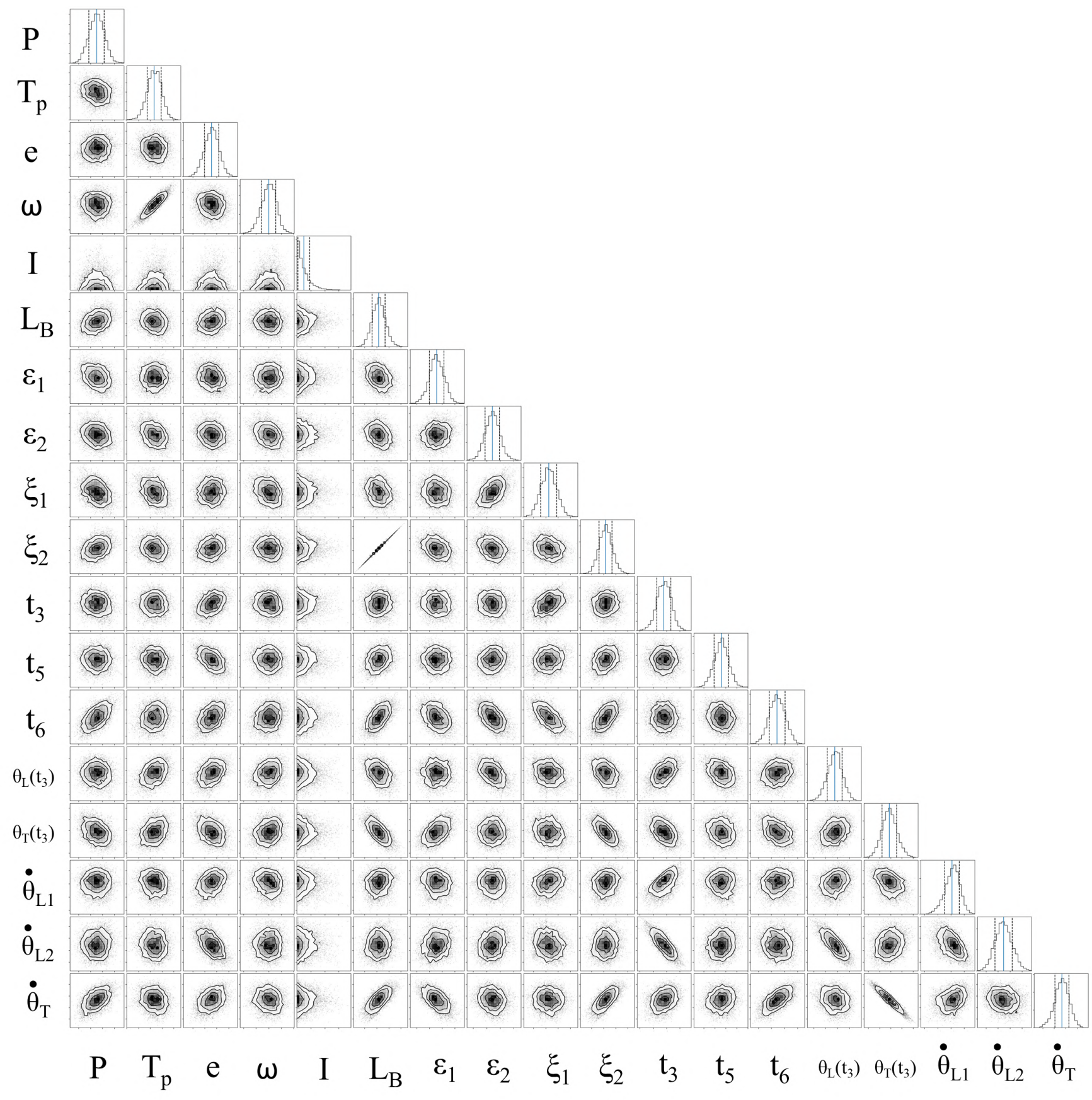

Figure B1. Two dimensional projection of the posterior probability distribution sampled using MCMC for $\alpha=0.5$. Blue solid lines indicate best fit values reported in Table 2, whereas black dashed lines indicate a $1 \sigma$ confidence interval. 